\title{
$\begin{array}{llllllllll}\mathbf{J} & \mathbf{o} & \mathbf{u} & \mathbf{r} & \mathbf{n} & \mathbf{a} & \mathbf{l} & & \mathbf{0} & \mathbf{f}\end{array}$ \\ Nonlinear Science
}

\section{Computation of Time-Periodic Solutions of the Benjamin-Ono Equation}

\author{
David M. Ambrose · Jon Wilkening
}

Received: 22 April 2008 / Accepted: 29 July 2009 / Published online: 5 February 2010

(C) The Author(s) 2010. This article is published with open access at Springerlink.com

\begin{abstract}
We present a spectrally accurate numerical method for finding nontrivial time-periodic solutions of nonlinear partial differential equations. The method is based on minimizing a functional (of the initial condition and the period) that is positive unless the solution is periodic, in which case it is zero. We solve an adjoint PDE to compute the gradient of this functional with respect to the initial condition. We include additional terms in the functional to specify the free parameters, which in the case of the Benjamin-Ono equation, are the mean, a spatial phase, a temporal phase, and the real part of one of the Fourier modes at $t=0$.

We use our method to study global paths of nontrivial time-periodic solutions connecting stationary and traveling waves of the Benjamin-Ono equation. As a starting guess for each path, we compute periodic solutions of the linearized problem by solving an infinite dimensional eigenvalue problem in closed form. We then use our
\end{abstract}

Communicated by M.I. Weinstein.

D.M. Ambrose current address: Department of Mathematics, Drexel University, Philadelphia, PA 19104. This work was supported in part by the National Science Foundation through grant DMS-0926378.

Work of J. Wilkening was supported in part by the Director, Office of Science, Computational and Technology Research, U.S. Department of Energy under Contract No. DE-AC02-05CH11231.

D.M. Ambrose ( $\varangle)$

Department of Mathematical Sciences, Clemson University, Clemson, SC 29634, USA

e-mail: ambrose@math.drexel.edu

J. Wilkening

Department of Mathematics and Lawrence Berkeley National Laboratory, University of California, Berkeley, CA 94720, USA

e-mail: wilken@math.berkeley.edu 
numerical method to continue these solutions beyond the realm of linear theory until another traveling wave is reached. By experimentation with data fitting, we identify the analytical form of the solutions on the path connecting the one-hump stationary solution to the two-hump traveling wave. We then derive exact formulas for these solutions by explicitly solving the system of ODEs governing the evolution of solitons using the ansatz suggested by the numerical simulations.

Keywords Periodic solutions · Benjamin-Ono equation · Nonlinear waves · Solitons · Bifurcation · Continuation · Optimal control · Adjoint equation · Spectral method

Mathematics Subject Classification (2000) $65 \mathrm{~K} 10 \cdot 37 \mathrm{M} 20 \cdot 35 \mathrm{Q} 53 \cdot 37 \mathrm{G} 15$

\section{Introduction}

A fundamental problem in the theory of ordinary and partial differential equations is to determine whether the equation possesses time-periodic solutions. Famous examples of ordinary differential equations with periodic solutions include the Brusselator (Field and Burger 1985; Govaerts 2000; Strogatz 2000) and the three-body problem (Arenstorf 1963; Hairer et al. 2000). In partial differential equations, time-periodic solutions can be "trivial" stationary or traveling waves, or can be genuinely timeperiodic. Such a problem can be studied in either the forced or unforced context. Forced problems include an external force in the PDE that is time-periodic; solutions with the same period are then sought. In the unforced problem, the period is one of the unknowns. In this work, we present a numerical method for finding genuinely time-periodic solutions of the unforced Benjamin-Ono equation with periodic boundary conditions. These solutions have many remarkable properties, which we will describe.

Our work is motivated by the calculations of Hou, Lowengrub, and Shelley for the vortex sheet with surface tension (Hou et al. 1994, 1997), and by the analysis of Plotnikov and Toland (2001), Iooss et al. (2005) for the water wave. Hou, Lowengrub, and Shelley developed an efficient numerical method to solve the initial value problem for the vortex sheet with surface tension. They performed calculations for a variety of initial conditions and values of the surface tension parameter, and found many situations in which the solutions appear to be close to time periodic. They did not, however, try to measure the deviation from time periodicity or attempt to vary the initial conditions to reduce this deviation. Plotnikov, Toland, and Iooss have proved the existence of time-periodic water waves, without surface tension, in the case of either finite or infinite depth. This is proved using a version of the Nash-Moser implicit function theorem. Their work includes no computation of the water waves. We aim to get a firmer handle on these solutions with an explicit calculation. To this end, in the present work, we develop a general numerical method for finding time-periodic solutions of nonlinear systems of partial differential equations and eventually plan to use this method for the vortex sheet and water wave problems. In fact, during the review process of the present work, we have succeeded in computing several families of time-periodic solutions of the vortex sheet with surface tension (Ambrose and Wilkening 2009). 
The Benjamin-Ono equation, developed in Benjamin (1967), Davis and Acrivos (1967), Ono (1975), is a model equation for the evolution of waves on deep water. It is a widely-studied dispersive equation, and much is known about solutions. It would be impossible to mention all results on Benjamin-Ono, but we mention, for example, that weak solutions exist for $u_{0} \in L^{2}$ (Saut 1979; Ginibre and Velo 1991), and that the solution exists for all time if $u_{0} \in H^{1}$ (Tao 2004). We chose the Benjamin-Ono equation as a first application for our numerical method because it is much less expensive to evolve than the vortex sheet or water wave, yet has many features in common with them, such as nonlocality (due to the Hilbert transform in the former case and the Birkhoff-Rott integral in the latter two cases.) In our numerical simulations, we find that the Benjamin-Ono equation has a rich family of nontrivial time-periodic solutions that act as rungs in a ladder connecting traveling waves with different speeds and wavelengths by creating or annihilating oscillatory humps that grow or shrink in amplitude until they become part of the stationary or traveling wave on the other side of the rung. The dynamics of these non-trivial solutions are often very interesting, sometimes resembling a low amplitude traveling wave superimposed on a larger carrier signal, and other times looking like a collection of interacting solitons that pass through each other or bounce off each other, depending on their relative amplitudes.

By fitting our numerical data, we have determined that all the solutions we have computed are special cases of the multiphase solutions studied by Case (1979), Satsuma and Ishimori (1979), Dobrokhotov and Krichever (1991), and Matsuno (2004), but with special initial conditions (that yield periodic orbits) and a modified mean (to change their speeds and allow bifurcations to occur between different levels of the hierarchy of multiphase solutions). We did not take advantage of this structure when we developed our numerical method; hence, our approach can also be used for nonintegrable problems. We also note that bifurcation within the family of multiphase solutions has not previously been discussed in the literature, nor has the remarkable dynamics of the Fourier coefficients of these solutions beyond the original derivation by Benjamin (1967) of the form of the traveling waves for this equation.

We are aware of very few works on the existence of time-periodic solutions for water wave model equations. Crannell has demonstrated (Crannell 1996) the existence of periodic, non-traveling, weak solutions of the Boussinesq equation using a generalization of the mountain pass lemma of Rabinowitz. Chen and Iooss have proved existence of time-periodic solutions in a two-way Boussinesq-type water wave model (Chen and Iooss 2005). As in Plotnikov and Toland (2001) and Iooss et al. (2005), there is no computation of the solution in either of these studies. Cabral and Rosa have recently discovered a period-doubling cascade of periodic solutions for a damped and forced version of the Korteweg-de Vries equation (Cabral and Rosa 2004). They use a Fourier pseudospectral method for the spatial discretization and a first order semiimplicit scheme in time. To find periodic solutions, they use a secant method on a numerical Poincaré map. Whereas our approach is based on minimizing a functional that measures deviation from periodicity, they rely on the stability of the orbit to converge to a periodic solution. They stop when they find a solution that returns to within one percent of its initial state, whereas we resolve our periodic solutions to 13-15 digits of accuracy, which allows us to study the analytic form of the solutions.

Water waves aside, many authors have investigated time-periodic solutions of other partial differential equations both numerically and analytically. For instance, 
Smiley proves existence of time-periodic solutions of a nonlinear wave equation on an unbounded domain (Smiley 1989); he also develops a numerical method for the same problem (Smiley 1990). On a finite domain, Brezis uses duality principles to prove the existence of periodic solutions of nonlinear vibrating strings in both the forced and unforced setting; see Brezis (1983). Mawhin has written a survey article on periodic solutions of semilinear wave equations (Mawhin 1995), which includes many references. Pao has developed a numerical method for the solution of timeperiodic parabolic boundary-value problems (Pao 2001). Pao gives various iterative schemes, but unlike the present work, these are not based on variational principles or the dual system. Brown et al. (1992) have used the popular software package AUTO (Doedel et al. 1991) to study Hopf bifurcation and loss of stability for traveling waves of the regularized Kuramoto-Sivashinsky equation. They observe modulated traveling waves similar to many of the solutions we have found for Benjamin-Ono. And of course, time-periodic solutions of systems of ordinary differential equations have also been widely studied, e.g., in Rabinowitz (1978, 1982), Zehnder (1983), Duistermaat (1984).

The most popular methods for the numerical solution of boundary value problems governed by ODEs, which include finding time-periodic solutions as a special case, are orthogonal collocation methods (Doedel et al. 1991) and shooting/multi-shooting methods (Stoer and Bulirsch 2002). These methods can also be used for PDEs via the method of lines, i.e., by discretizing space first to obtain an ODE. Orthogonal collocation methods (such as implemented in AUTO) require a nonlinear system of equations to be solved involving all the degrees of freedom at every timestep. In many of our simulations, the solution at every timestep cannot even be stored simultaneously in memory; hence, it would be impossible to solve a system of equations for all these variables. For PDEs, shooting methods are also very expensive as the Jacobian of the functional measuring errors in the boundary conditions must be computed with respect to variation of the $N$ "unknown" initial conditions, which is $N$ times more expensive than computing the functional itself.

Lust and Roose (1996) propose an interesting solution to this problem in which a shooting method is used for some of the degrees of freedom while iteration on a Poincaré map is used for the other degrees of freedom. They aim to exploit the fact that many high dimensional systems actually exhibit low-dimensional dynamical behavior. In the present work, we offer an alternative strategy based on the following idea: while the Jacobian of the residual measuring error in each of the boundary conditions is expensive to compute, the gradient of the sum of squares of this residual can be computed by solving a single adjoint PDE. Although the full Jacobian gives more information, it is $N$ times more expensive to compute. We find that it is more efficient to use a quasi-Newton method with only the gradient information than it is to use a full Newton method with the Jacobian. This approach is not restricted to finding time-periodic solutions; it will work for any boundary value problem. Also, although we have not tried it, our method can be easily adapted to incorporate the idea behind multishooting methods, namely that by introducing "interior" initial conditions and boundary conditions, we can avoid a great deal of the ill conditioning due to nearby trajectories diverging exponentially in time.

The closest numerical method to our own that we have found is due to Bristeau et al. (1998), who developed a least squares shooting method for numerical computation 
of time-periodic solutions of linear dynamical systems with applications in scattering phenomena in two and three dimensions; see also Glowinski and Rossi (2006). These authors employ methods of control theory to compute variational derivatives, and although they only apply their methods to linear problems, they mention that their techniques will also work on non-linear problems. Our method can be considered an extension of their approach that focuses on the difficulties that arise due to nonlinearity. In particular, we replace their conjugate gradient solver with a blackbox minimization algorithm, (the BFGS method (Nocedal and Wright 1999)), and include an additional penalty function to prescribe the values of the free parameters that describe the manifold of nontrivial time-periodic solutions. Without this penalty function, the basic method is only found to produce constant solutions and traveling waves.

This paper is organized as follows: In Sect. 2, we discuss spatially periodic stationary and traveling solutions of the Benjamin-Ono equation, the bifurcations from constant solutions to traveling waves, and the pole dynamics of meromorphic solutions. In Sect. 3, we investigate time-periodic solutions of the linearized BenjaminOno equation; this is the linearization about the stationary solutions discussed previously. To analyze the linearized problem, we compute (numerically) the spectrum and eigenfunctions of the relevant linear operator and deduce their analytic form by trial and error; the resulting formulas can be verified rigorously (but we omit details).

In Sect. 4, we describe our numerical method, which involves minimizing a nonnegative functional that is zero if and only if the solution is periodic. We solve an adjoint PDE to compute the variational derivative of this functional with respect to perturbation of the initial condition and use the BFGS minimization algorithm to minimize the functional. The Benjamin-Ono and adjoint equations are solved with a pseudo-spectral collocation method using a fourth order, semiimplicit Runge-Kutta scheme. We use a penalty function to rule out constant solutions and traveling waves, and to prescribe the free parameters of the manifold of nontrivial solutions. We then vary a bifurcation parameter to study the global properties of these nontrivial solutions. In the present work, we apply this method only to the Benjamin-Ono equation, but we are confident that this method is applicable to virtually any system of partial differential equations that possesses time-periodic solutions.

In Sect. 5, we use our method to study the global behavior of nontrivial timeperiodic solutions far beyond the realm of validity of the linearization about stationary and traveling waves. We will follow one such path to discover that the one-hump stationary solution is connected to the two-hump traveling wave by a path of non-trivial time periodic solutions. In Sect. 6, we reformulate the ODE governing the evolution of poles to reveal an exact formula for the solutions on the path studied numerically in Sect. 5. Thus, unexpectedly, we have proved that nontrivial time-periodic solutions bifurcate from stationary solutions by exhibiting a family of them explicitly. In a follow-up paper (Ambrose and Wilkening 2009), we will classify all bifurcations from traveling waves, study the paths of nontrivial solutions connecting several of them, propose a conjecture explaining how they all fit together, and describe their analytic form to the extent that we are able. We end with a few concluding remarks in Sect. 7. 


\section{Stationary, Traveling, and Soliton Solutions}

We consider the spatially periodic Benjamin-Ono equation with the following sign convention:

$$
u_{t}=H u_{x x}-u u_{x} .
$$

Of course, the operator $H$ is the Hilbert transform. Recall that the symbol of $H$ is $\hat{H}(k)=-i \operatorname{sgn}(k)$. Many authors include a factor of two in the convection term and place a minus sign in front of $H$. The former change causes solutions to be scaled by a factor of $1 / 2$ while the latter change has no effect as $H$ is defined with the opposite sign with this convention.

This equation possesses a two-parameter family of stationary solutions, namely

$$
u(x)=\frac{1-3 \beta^{2}}{1-\beta^{2}}+\frac{4 \beta[\cos (x-\theta)-\beta]}{1+\beta^{2}-2 \beta \cos (x-\theta)} \quad(-1<\beta<1, \theta \in \mathbb{R}) .
$$

These solutions have mean $\alpha$, related to $\beta$ via

$$
\alpha(\beta)=\frac{1-3|\beta|^{2}}{1-|\beta|^{2}}, \quad|\beta|^{2}=\frac{1-\alpha}{3-\alpha} .
$$

Changing the sign of $\beta$ is equivalent to the phase shift $\theta \rightarrow \theta-\pi$. It is convenient to complexify $\beta$ and define $u_{\beta}$ to be the mean-zero part of (2) with $\beta \rightarrow|\beta|, \theta \rightarrow \arg \bar{\beta}$ :

$$
\text { stationary solution }=\alpha(\beta)+u_{\beta}(x), \quad \beta=|\beta| e^{-i \theta} \in \Delta=\{z \in \mathbb{C}:|z|<1\} .
$$

Note that the subscript $\beta$ does not indicate a derivative here. Several stationary solutions with $\beta$ real and negative are shown in Fig. 1. The Fourier representation of $u_{\beta}$ is simply

$$
\hat{u}_{\beta, k}=\left\{\begin{array}{ll}
2 \bar{\beta}^{|k|}, & k<0 \\
0, & k=0 \\
2 \beta^{k}, & k>0
\end{array}\right\}
$$

where $\bar{\beta}$ is the complex conjugate of $\beta$. These functions $u_{\beta}(x)$ are the building blocks for the meromorphic solutions discussed below.

Note that the constant solution $u \equiv \alpha_{0}$ is also a stationary solution, as are the rescaled solutions

$$
u_{N, \beta}(x)=N \alpha(\beta)+N u_{\beta}(N x) \quad(\beta \in \Delta, N=1,2,3, \ldots),
$$

which have mean $\alpha_{0}=N \alpha(\beta)$. If we restrict attention to stationary solutions with even symmetry (i.e., with $\beta$ real), we find that there is a pitchfork bifurcation at each positive integer (using the mean as a bifurcation parameter). As $\alpha_{0}$ changes from $N^{+}$to $N^{-}$, the constant solution splits, yielding two additional ( $N$-hump stationary) solutions, namely $u_{N, \beta}(x)$ with $\beta=0^{ \pm}$. The pitchfork would be obtained by plotting the real part of the $N$ th Fourier mode versus the mean, where we observe that the 


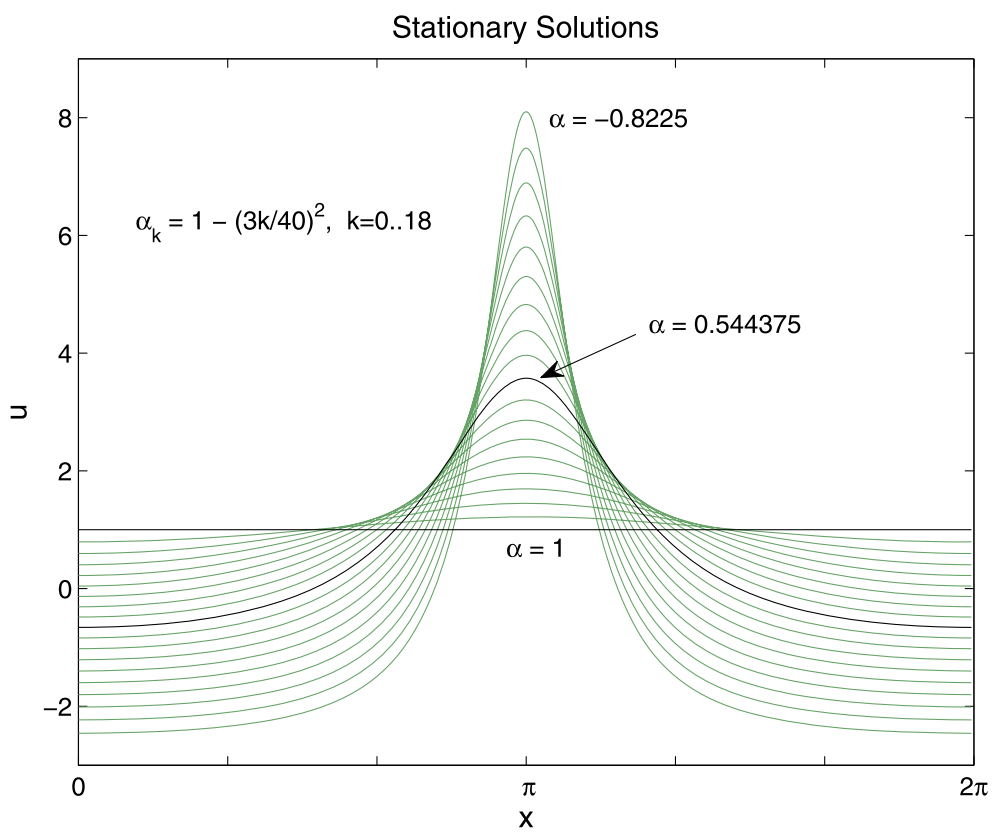

Fig. 1 Stationary solutions of the Benjamin-Ono equation

Fourier representation of $u=u_{N, \beta}$ (for any $\beta \in \Delta$ ) is given by

$$
\hat{u}_{k}= \begin{cases}N \alpha(\beta), & k=0, \\ 2 N \beta^{k / N}, & k \in N \mathbb{Z}, k>0, \\ 2 N \bar{\beta}^{|k| / N}, & k \in N \mathbb{Z}, k<0, \\ 0 & \text { otherwise. }\end{cases}
$$

If we do not restrict attention to even solutions, the phase shift $\theta$ acts as a second parameter connecting the two outer branches of the pitchfork into a two-dimensional, bowl-shaped sheet (plotting the real and imaginary parts of the $N$ th Fourier mode versus the mean).

In the bifurcation problem just described, we varied the mean $\alpha_{0}$ and found bifurcations from constant solutions to stationary solutions at the positive integers. The remainder of this paper deals with bifurcation from these stationary solutions to nontrivial time-periodic solutions and their global continuation beyond the realm of linear theory. Rather than varying the mean, we will hold $\alpha_{0} \in \mathbb{R}$ constant and use another quantity (such as the period $T$ or the real part of one of the Fourier modes of $u$ at $t=0$ ) as the bifurcation parameter. As a first step, let us consider bifurcation from constant solutions to traveling waves holding $\alpha_{0} \in \mathbb{R}$ constant and varying $T$.

All traveling wave solutions of the Benjamin-Ono equation can be found by applying a simple transformation to a stationary solution, and vice versa. Indeed, if 
$u(x, t)$ is any solution of $(1)$, then

$$
U(x, t)=u(x-c t, t)+c
$$

is also a solution; thus, adding a constant $c$ to a stationary solution causes it to travel to the right with speed $c$. We can parametrize these $N$-hump traveling waves by their mean $\alpha_{0} \in \mathbb{R}$ and decay/phase parameter $\beta \in \Delta$ :

$$
u_{\alpha_{0}, N, \beta}(x, t)=u_{N, \beta}(x-c t)+c \quad\left(c=\alpha_{0}-N \alpha(\beta)\right) .
$$

If we express the period $T=2 \pi /(N|c|)$ in terms of $\beta$ and solve for $\beta$, we find that we can bifurcate from any constant solution $u \equiv \alpha_{0}$ to an $N$-hump traveling solution with the same mean. If $\alpha_{0}<N$, a pitchfork from the constant solution occurs at $T_{0}=$ $2 \pi /\left[N\left(N-\alpha_{0}\right)\right]$; as we increase $T$ from $T_{0}$ to $\infty, \alpha=\left[2 \pi /(N T)+\alpha_{0}\right] / N$ decreases from 1 to $\alpha_{0} / N$ and $|\beta|$ varies from 0 to $\sqrt{\left(1-\alpha_{0} / N\right) /\left(3-\alpha_{0} / N\right)}$. Similarly, if $\alpha_{0}>N$, a pitchfork occurs at $T_{0}=2 \pi /\left[N\left(\alpha_{0}-N\right)\right]$; as we decrease $T$ from $T_{0}$ to $0, \alpha=\left[\alpha_{0}-2 \pi /(N T)\right] / N$ decreases from 1 to $-\infty$, and $|\beta|$ varies from 0 to 1 . And if $\alpha_{0}=N$, the situation is qualitatively similar to the latter case, but the pitchfork occurs at $T_{0}=\infty$, i.e., all three solutions (with $\beta$ real) exist for any period $T>0$.

We remark that if the traveling waves described above have zero mean, they are a special case of the meromorphic $N$-particle "periodic soliton" solutions described in Case (1978), namely

$$
u(x, t)=2 \operatorname{Re}\left\{\sum_{l=1}^{N} \frac{2}{e^{i\left[x+t-x_{l}(t)\right]}-1}\right\},
$$

where $\operatorname{Im}\left\{x_{l}(0)\right\}>0$ and the $x_{l}(t)$ satisfy the system of differential equations

$$
\frac{d x_{l}}{d t}=\sum_{\substack{m=1 \\ m \neq l}}^{N} \frac{2}{e^{-i\left(x_{m}-x_{l}\right)}-1}+\sum_{m=1}^{N} \frac{2}{e^{-i\left(x_{l}-\bar{x}_{m}\right)}-1} \quad(1 \leq l \leq N) .
$$

In our notation, we write

$$
x_{l}(t)=\overline{i \log \beta_{l}(t)}=\theta_{l}(t)-i \log \left|\beta_{l}(t)\right| \quad\left(\beta_{l}=\left|\beta_{l}\right| e^{-i \theta_{l}}=e^{-i \bar{x}_{l}}\right)
$$

and generalize to the case that the mean $\alpha_{0}$ can be nonzero. We find from (9) and (7) that

$$
u(x, t)=\alpha_{0}+\sum_{l=1}^{N} u_{\beta_{l}(t)}(x)
$$

is a solution of (1) if the variables $\beta_{l} \in \Delta$ satisfy

$$
\dot{\beta}_{l}=\sum_{\substack{m=1 \\ m \neq l}}^{N} \frac{-2 i \beta_{l}^{2}}{\beta_{l}-\beta_{m}}+\sum_{m=1}^{N} \frac{2 i \beta_{l}^{2}}{\beta_{l}-\bar{\beta}_{m}^{-1}}+i\left(2 N-1-\alpha_{0}\right) \beta_{l} \quad(1 \leq l \leq N) .
$$


The $N$-hump traveling wave then has the representation

$$
u_{\alpha_{0}, N, \beta}(x, t)=\alpha_{0}+\sum_{l=1}^{N} u_{\beta_{l}(t)}(x), \quad \beta_{l}(t)=\sqrt[N]{\beta} e^{-i c t}, c=\alpha_{0}-N \alpha(\beta)
$$

where each $\beta_{l}$ is assigned a distinct $N$ th root of $\beta$. As we are interested in developing numerical methods that generalize to more complicated systems such as the vortex sheet with surface tension and the water wave, we do not exploit the existence of meromorphic solutions in our numerical method; however, the nontrivial time-periodic solutions we find do turn out to be of this form; see Sect. 6.

\section{Linear Theory}

We formulate the problem of finding time-periodic solutions of the Benjamin-Ono equation as that of finding an initial condition $u_{0}$ and period $T$ such that $F\left(u_{0}, T\right)=$ 0 , where $F: H^{1} \times \mathbb{R} \rightarrow H^{1}$ is given by

$$
F\left(u_{0}, T\right)=u(\cdot, T)-u_{0}, \quad u_{t}=H u_{x x}-u u_{x}, \quad u(\cdot, 0)=u_{0} .
$$

Clearly, stationary solutions are periodic with any period $T$. In this section, we linearize $F$ about these stationary solutions and use solutions of the linearized problem as initial search directions to find time-periodic solutions of the nonlinear problem. Bifurcation from traveling waves can be reduced to this case by adding an appropriate constant and requiring that the period of the perturbation coincide with the period of the traveling wave (although there may be a phase shift involved as well). We present a detailed analysis of the traveling case in Ambrose and Wilkening (2009).

\subsection{Linearization About Stationary Solutions}

Let $u=u_{N, \beta}$ be an arbitrary $N$-hump stationary solution. If $u(x)+v(x, t)$ is to satisfy (1) to first order in $v$, then $v$ should satisfy

$$
v_{t}=H v_{x x}-(u v)_{x} .
$$

(The exact solution satisfies $v_{t}=H v_{x x}-(u v)_{x}-v v_{x}$. .) Equation (13) can be written

$$
v_{t}=i B A v,
$$

where the (unbounded, self-adjoint) operators $A$ and $B$ on $H^{1}$ are defined as

$$
A=H \partial_{x}-u, \quad B=\frac{1}{i} \partial_{x} .
$$

To solve (14), we are interested in the eigenvalue problem

$$
B A z=\omega z,
$$


so that if $B A$ has a complete set of eigenvectors, the general solution of (14) will be a superposition of functions of the form

$$
v(x, t)=\operatorname{Re}\left\{C z(x) e^{i \omega t}\right\}, \quad C \in \mathbb{C} .
$$

Of course, the eigenvalues of a composition of Hermitian operators need not be real, but for $A$ and $B$ in (15), we can compute all the eigenvalues explicitly, and they are indeed real. We do this numerically (which surprisingly leads us to formulas we can check analytically) by truncating the Fourier representations of $A$ and $B$ and computing the eigenvalues of the matrix $\hat{B} \hat{A}$. More precisely, we choose a cutoff frequency $K$ (e.g., $K=240)$ and define the $(2 K-1) \times(2 K-1)$ matrices

$$
\hat{A}_{k l}=|k| \delta_{k l}-\hat{u}_{k-l}=|k| \delta_{k l}-\overline{\hat{u}_{l-k}}, \quad \hat{B}_{k l}=k \delta_{k l} \quad(-K<k, l<K),
$$

where $\hat{u}_{k}$ was given in (6) and $\delta_{k l}=1$ if $k=l$ and 0 otherwise. By carefully studying the eigenvalues for different values of $N$ and $\beta=-\sqrt{(1-\alpha) /(3-\alpha)}$ with $\alpha<1$, we determined that

$$
\omega_{N, n}= \begin{cases}-\omega_{N,-n}, & n<0, \\ 0, & n=0, \\ (n)(N-n), & 1 \leq n \leq N-1, \\ (n+1-N)(n+1+N(1-\alpha)), & n \geq N .\end{cases}
$$

With this numbering, the first $N-1$ nonzero eigenvalues are independent of $\alpha$ :

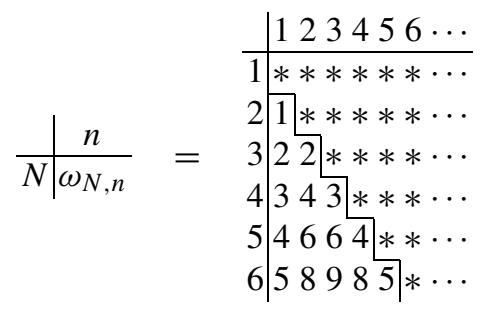

Note that $\omega_{N, N}=(2-\alpha) N+1 \geq N+1$ and $\omega_{N, n}$ is strictly increasing in $n$ for $n \geq N$, but $\omega_{N, N}$ could be less than $\omega_{N,\lfloor N / 2\rfloor}$ when $N \geq 6$ (and some of the eigenvalues can coalesce, increasing their multiplicity). Nevertheless, the ordering of the eigenvalues in (18) is more convenient than the monotonic ordering due to the fact that a pathway of non-trivial solutions connecting an $N$-hump traveling wave to an $N^{\prime}$-hump traveling wave with $N<N^{\prime}$ seems to involve $\omega_{N, n}$ and $\omega_{N^{\prime}, n^{\prime}}$ with $n \geq N$ and $n^{\prime}<N^{\prime}$ satisfying $N^{\prime}=n+1$ and $n^{\prime}=N^{\prime}-N$ (see Ambrose and Wilkening 2009.) These global reconnection formulas would be much more complicated if the eigenvalues were ordered monotonically.

The zero eigenvalue $\omega_{N, 0}=0$ has geometric multiplicity two and algebraic multiplicity three. The fact that the dimension of the kernel is independent of $\alpha$ indicates that there are no special values of the mean $N \alpha$ at which these $N$-hump stationary solutions bifurcate to more complicated stationary solutions. The two eigenfunctions 
in the kernel of $B A$ are

$$
z_{N, 0}^{(1,0)}(x)=-u_{x}(x)=\left.\frac{\partial}{\partial \theta}\right|_{\theta=0} u_{N, \beta}(x-\theta), \quad z_{N, 0}^{(2)}(x)=\frac{\partial}{\partial|\beta|} u_{N, \beta}(x),
$$

which correspond to translating the stationary solution by a phase or decreasing its mean, $N \alpha=N\left(1-3|\beta|^{2}\right) /\left(1-|\beta|^{2}\right)$. There is also a Jordan chain (Wilkening 2007) of length two associated with $z_{N, 0}^{(1,0)}(x)$, namely

$$
z_{N, 0}^{(1,1)}(x)=i \quad\left(B A z_{N, 0}^{(1,1)}=z_{N, 0}^{(1,0)}\right) .
$$

The corresponding solution of (14) is

$$
v(x, t)=-i z_{N, 0}^{(1,1)}(x)+t z_{N, 0}^{(1,0)}(x)=1-t u_{x}(x)=\left.\frac{\partial}{\partial \varepsilon}\right|_{\varepsilon=0}[u(x-\varepsilon t)+\varepsilon],
$$

i.e., this linear growth mode arises due to the fact that adding a constant to a stationary solution causes it to travel. The multiple eigenvalues $\omega_{N, n}=\omega_{N, N-n}$ with $1 \leq n \leq$ $N-1$ pose a minor obstacle to obtaining explicit formulas for the eigenvectors. We eventually realized that because the shift operator

$$
S_{\theta} z(x)=z(x-\theta), \quad \hat{S}_{\theta, k l}=e^{-i k \theta} \delta_{k l}, \quad \theta=2 \pi / N
$$

commutes with $B A$, the eigenspaces of $B A$ are invariant under the action of $S_{\theta}$. Thus, we can impose the additional requirement that if $z$ is an eigenvector of $B A$ corresponding to a multiple eigenvalue, then $z$ should also satisfy

$$
\hat{z}_{k} \neq 0 \quad \text { and } \quad \hat{z}_{l} \neq 0 \quad \Rightarrow \quad k-l \in N \mathbb{Z},
$$

i.e. the nonzero Fourier coefficients are equally spaced with stride length $N$. Using this condition to make the eigenvectors unique up to scaling, we were able to recognize the patterns that emerge in the numerical eigenvectors (with the exception of the coefficient $C$ and the $j=0$ case when $n \geq N$, which we determined analytically):

$$
\begin{aligned}
& \left.\hat{z}_{N, n, k}\right|_{k=n+j N}=\left\{\begin{array}{ll}
\left(1+\frac{N(|j|-1)}{N-n}\right) \bar{\beta}^{|j|-1}, & j<0 \\
C\left(1+\frac{N j}{n}\right) \beta^{j+1}, & j \geq 0
\end{array}\right\} \quad\left(\begin{array}{c}
1 \leq n \leq N-1, \\
C=\frac{-n N}{(N-n)\left[n+(N-n)|\beta|^{2}\right]}
\end{array}\right), \\
& \left.\hat{z}_{N, n, k}\right|_{k=n-N+1+j N}=\left\{\begin{array}{ll}
0, & j<0 \\
\frac{-\bar{\beta}}{\left(1-|\beta|^{2}\right)^{2}}\left[1-\left(1-\frac{N}{n+1}\right)|\beta|^{2}\right], & j=0 \\
\left(1+\frac{N(j-1)}{n+1}\right) \beta^{j-1}, & j \geq 1
\end{array}\right\} \quad(n \geq N) .
\end{aligned}
$$

These formulas can be summed to obtain $z_{N, n}(x)$ as a rational function of $e^{i x}$, but we prefer to work with the Fourier coefficients. Note that as $n \rightarrow \infty$ (holding $N$ fixed), the index $k=n-N+1$ of the first nonzero Fourier mode increases to infinity. The eigenvectors corresponding to negative eigenvalues $\omega_{N,-n}$ with $n \geq 1$ satisfy $z_{N,-n}(x)=\overline{z_{N, n}(x)}$, so the Fourier coefficients appear in reverse order, conjugated: $\hat{z}_{N,-n, k}=\overline{\hat{z}_{N, n,-k}}$. When $\beta$ is real, the Fourier coefficients are real and 
$z_{N,-n}(x)=z_{N, n}(-x)$. We have verified the formulas (18) and (24) analytically, and can also prove that the Fourier representation of these eigenvectors (together with the associated vector corresponding to the Jordan chain) form a Riesz basis for $\ell^{2}(\mathbb{Z})$; hence, we have not missed any eigenvalues.

\subsection{Bifurcation from Stationary Solutions}

Now that we have solved the eigenvalue problem for $B A$, we can compute the derivative of the operator $F$ in (12) above. We continue to assume that $u$ is an $N$-hump stationary solution so that $D F=\left(D_{1} F, D_{2} F\right): H^{1} \times \mathbb{R} \rightarrow H^{1}$ satisfies

$$
\begin{aligned}
& D_{1} F(u, T) v_{0}=\left.\frac{\partial}{\partial \varepsilon}\right|_{\varepsilon=0} F\left(u+\varepsilon v_{0}, T\right)=v(\cdot, T)-v_{0}=\left[e^{i B A T}-I\right] v_{0}, \\
& D_{2} F(u, T) \tau=\left.\frac{\partial}{\partial \varepsilon}\right|_{\varepsilon=0} F(u, T+\varepsilon \tau)=0 .
\end{aligned}
$$

Note that $v_{0} \in \operatorname{ker} D_{1} F(u, T)$ iff the solution $v(x, t)$ of the linearized problem is periodic with period $T$. These solutions of the linearized problem serve as initial search directions in which to find periodic solutions of the non-linear problem. Since $D_{2} F=0$ in the stationary case, the nullspace $\mathcal{N}$ of $D F=\left(D_{1} F, D_{2} F\right)$ is of the form $\mathcal{N}=\mathcal{N}_{1} \times \mathbb{R}$ where $\mathcal{N}_{1}=\operatorname{ker} D_{1} F$. A basis for $\mathcal{N}_{1}$ consists of the functions

$$
v_{0}(x)=\operatorname{Re}\left\{z_{N, n}(x)\right\} \quad \text { and } \quad v_{0}(x)=\operatorname{Im}\left\{z_{N, n}(x)\right\},
$$

where $n$ ranges over all integers such that

$$
\omega_{N, n} T \in 2 \pi \mathbb{Z}
$$

Negative values of $n$ have already been accounted for in (26) using $z_{N,-n}(x)=$ $\overline{z_{N, n}(x)}$. The $n=0$ case always yields two vectors in the kernel, namely those in (20). These directions do not cause bifurcations as they lead to other stationary solutions in the two parameter family. Thus, the periods at which bifurcations are expected are

$$
T_{N, n, m}=\frac{2 \pi m}{\omega_{N, n}} \quad(m, n \geq 1) .
$$

Note that this set is dense on the positive real line since $\omega_{N, n} \rightarrow \infty$ as $n \rightarrow \infty$. This leads to a small divisor problem when trying to apply the Liapunov-Schmidt reduction (Golubitsky and Schaeffer 1985; Kielhöfer 2004) to rigorously analyze bifurcations in the set of solutions of the equation $F\left(u_{0}, T\right)=0$. For other problems, small divisors have been dealt with successfully using Nash-Moser theory; see, e.g. Nirenberg (2001), Plotnikov and Toland (2001), Iooss et al. (2005). As most of the bifurcations responsible for the small divisor problem involve high frequency perturbations that are smoothed out by the numerical discretization, we have not found small divisors to cause major difficulties in our ability to track paths of time-periodic solutions.

In our numerical studies, we have found that each of the eigenfunctions $z_{N, n}(x)$ with $n \geq 1$ gives a direction along which a sheet of nontrivial solutions bifurcates 
Fig. 2 First seven bifurcations from the constant solution $u(x)=\alpha_{0}$ to traveling waves with $n$-humps. The period shown is

$T=2 \pi m /\left[n\left(n \alpha-\alpha_{0}\right)\right]$ with $m=2 n(n-1 / 2)$. We used this to solve for $|\beta|$ in terms of $T$ via (3). The amplitude shown is the difference between the maximum and minimum values of the solution, i.e., $8 n|\beta| /\left(1-|\beta|^{2}\right)$

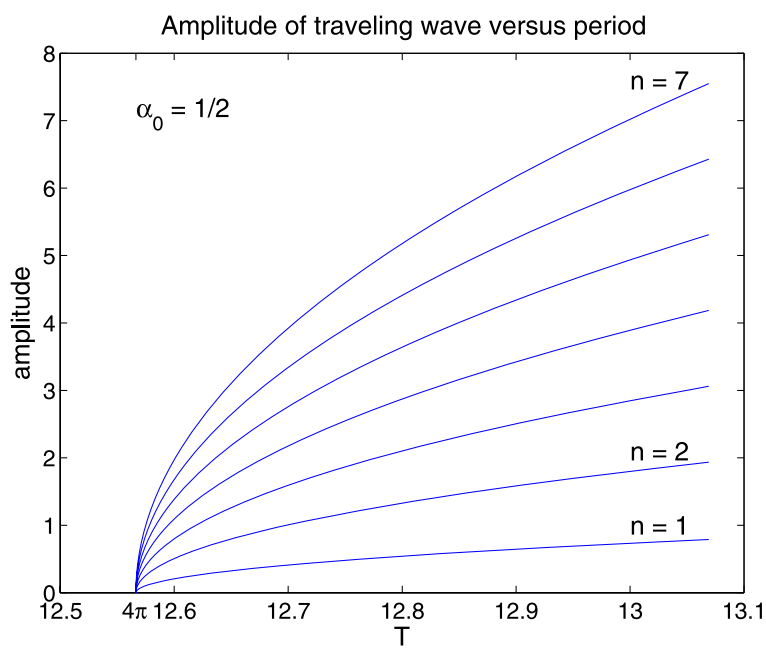

from the $N$-hump stationary solution. More precisely, if we let a parameter $\varepsilon \rightarrow 0$, there appear to be time-periodic solutions of the nonlinear problem with period $T_{\varepsilon}=$ $T_{N, n, m}+O\left(\varepsilon^{2}\right)$ that agree with the real and imaginary parts of $\varepsilon z_{N, n}(x)$ at $t=0$ and $t=-T_{\varepsilon} /(4 m)$, respectively, to $O\left(\varepsilon^{2}\right)$. This is interesting because in cases that several (even infinitely many) of the eigenfunctions $z_{N, n}(x)$ lead to solutions of the linearized problem with the same period, most linear combinations of these solutions will not give a bifurcation direction. In other words, the eigenfunctions $z_{N, n}(x)$ are already diagonalized with respect to the nonlinear effects of the bifurcation. For this to work out, it is essential that when $\omega_{N, n}$ is a multiple eigenvalue, $z_{N, n}(x)$ is chosen as in (23) to simultaneously diagonalize the shift operator $S_{2 \pi / N}$.

We observe an analogous phenomenon when bifurcating from constant solutions to traveling waves; see Fig. 2. When $u=\alpha_{0}$ is a constant function in (15) above, $\hat{A}$ and $\hat{B}$ are both diagonal matrices, the eigenvalues and eigenvectors of $B A$ are given by

$$
\omega_{n}=n\left(|n|-\alpha_{0}\right), \quad z_{n}(x)=e^{i n x} \quad(n \in \mathbb{Z}),
$$

and the bifurcation times are given by

$$
T_{n, m}=2 \pi m /\left(n\left|\alpha_{0}-n\right|\right) \quad(n, m \geq 1) .
$$

Note that in this simplified problem, the bifurcation index $n$ turns out to be the number of humps. If $\alpha_{0}=1 / 2$ and $T=4 \pi$, then $\omega_{n} T \in 2 \pi \mathbb{Z}$ for every $n$, i.e., the kernel $\mathcal{N}_{1}$ of $D_{1} F(u, T)$ is the whole space $H^{1}$. Nevertheless, the traveling solutions that emerge from this bifurcation are no different than if $\alpha_{0}$ were irrational and $\mathcal{N}_{1}$ were spanned by $\{1, \cos n x, \sin n x\}$ for some fixed $n$-they all just happen to join together at $T=4 \pi$ for this value of the mean. More specifically, the $n$-hump traveling solutions $u_{\alpha_{0}, n, \beta}(x, t)$ defined in (8) above have the property that as $\beta \rightarrow 0$ (and hence $\alpha \rightarrow 1)$, a multiple $m$ of their shortest period $2 \pi /\left[n\left(n \alpha-\alpha_{0}\right)\right]$ converges to $4 \pi$. So while $\cos x$ and $\cos 2 x$ are each bifurcation directions for initial conditions that lead to periodic solutions of the nonlinear problem, $(\cos x+\cos 2 x)$ is not. 


\section{The Method}

In order to compute nontrivial time periodic solutions, we define the functional

$$
G_{\text {tot }}\left(u_{0}, T\right)=G\left(u_{0}, T\right)+\varphi\left(u_{0}, T\right)
$$

with

$$
G\left(u_{0}, T\right)=\frac{1}{2} \int_{0}^{2 \pi}\left[u(x, T)-u_{0}(x)\right]^{2} d x
$$

and look for minimizers of $G_{\text {tot }}$ with the hope that the minimum value will be zero. Here, $\varphi\left(u_{0}, T\right)$ is a nonnegative penalty function designed to eliminate spatial and temporal phase shifts and specify the mean $\alpha_{0}$ and the amplitude of one of the Fourier modes at $t=0$. Our first goal is to find an efficient method of computing the variational derivative of $G$. As usual in optimal control problems (Pironeau 1984), there is an adjoint PDE that allows us to compute $\frac{\delta G}{\delta u_{0}}$ in as little time as it takes to compute $G$ itself. We will then use a spectral method in space and a fourth order semiimplicit Runge-Kutta method (Cooper and Sayfy 1983; Kennedy and Carpenter 2003; Wilkening 2007) in time to solve the Benjamin-Ono and adjoint equations to compute $G, \frac{\delta G}{\delta u_{0}}$ and $\frac{\partial G}{\partial T}$ in the inner loop of the BFGS minimization algorithm (Broyden 1970; Nocedal and Wright 1999).

\subsection{Variational Derivative of $G$}

Let $u_{0}$ be any function in $H^{1}$ (not necessarily leading to a periodic solution). Evidently,

$$
\frac{\partial}{\partial T} G\left(u_{0}, T\right)=\int_{0}^{2 \pi}\left[u(x, T)-u_{0}(x)\right] u_{t}(x, T) d x .
$$

Let $v_{0} \in H^{1}$ be given and define $\dot{G}=D_{1} G\left(u_{0}, T\right) v_{0}$, i.e.,

$$
\dot{G}=\left.\frac{d}{d \varepsilon}\right|_{\varepsilon=0} G\left(u_{0}+\varepsilon v_{0}, T\right)=\int_{0}^{2 \pi}\left[u(x, T)-u_{0}(x)\right]\left[v(x, T)-v_{0}(x)\right] d x .
$$

Here, $v(x, t)=\dot{u}(x, t)=\left.\frac{d}{d \varepsilon}\right|_{\varepsilon=0} u(x, t, \varepsilon)$ with $u(x, t, \varepsilon)$ the solution of BenjaminOno with initial condition $u(x, 0, \varepsilon)=u_{0}(x)+\varepsilon v_{0}(x)$. We can compute $v$ by solving the variational equation

$$
v_{t}=H v_{x x}-(u v)_{x}, \quad v(x, 0)=v_{0}(x),
$$

which is linear but nonautonomous (as $u$ depends on time in general). Our next task is to eliminate $v(x, T)$ from (32) and represent $\dot{G}$ as an inner product:

$$
\dot{G}=\int_{0}^{2 \pi} \frac{\delta G}{\delta u_{0}}(x) v_{0}(x) d x
$$


The idea is to define a function $w(x, s)$ going backward in time (with $s=T-t$ ) such that

$$
w(x, 0)=w_{0}(x)=u(x, T)-u_{0}(x)
$$

and then determine how $w$ should evolve so that

$$
\int_{0}^{2 \pi} w(x, 0) v(x, T) d x=\int_{0}^{2 \pi} w(x, T) v(x, 0) d x .
$$

Let us define the solution operator $V\left(t_{2}, t_{1}\right): H^{1} \rightarrow H^{1}$ for the linearized equation (33) as the mapping that evolves an initial condition specified at time $t_{1}$ to the solution at time $t_{2}$. These operators satisfy a nonautonomous, time reversible version of familiar semigroup properties:

$$
V\left(t_{1}, t_{1}\right)=I, \quad V\left(t_{3}, t_{1}\right)=V\left(t_{3}, t_{2}\right) V\left(t_{2}, t_{1}\right) \quad\left(t_{1}, t_{2}, t_{3} \in \mathbb{R}\right) .
$$

Equation (36) may now be written

$$
\left\langle w_{0}, V(T, 0) v_{0}\right\rangle=\left\langle W(T, 0) w_{0}, v_{0}\right\rangle
$$

where $\langle\cdot, \cdot\rangle$ is the $L^{2}$ inner product and we define $W\left(s_{2}, s_{1}\right)=V\left(t_{1}, t_{2}\right)^{*}$ with $t_{j}=$ $T-s_{j}$. It follows from (37) that $W\left(s_{1}, s_{1}\right)=I$ and $W\left(s_{3}, s_{1}\right)=W\left(s_{3}, s_{2}\right) W\left(s_{2}, s_{1}\right)$. What remains is to determine how this nonautonomous semigroup $W$ is generated. Taking the inner product of $v_{t}$ with $w$, we have

$$
\begin{aligned}
\int v_{t}(x, t) w(x, s) d x & =\lim _{h \rightarrow 0} \int\left(\left[\frac{V(t+h, t)-V(t, t)}{h}\right] v(x, t)\right) w(x, s-h) d x \\
& =\lim _{h \rightarrow 0} \int v(x, t)\left(\left[\frac{W(s, s-h)-I}{h}\right] w(x, s-h)\right) d x \\
& =\int v(x, t) w_{s}(x, s) d x .
\end{aligned}
$$

We learn that

$$
\int v w_{s} d x=\int v_{t} w d x=\int\left[H v_{x x}-(u v)_{x}\right] w d x=\int v\left[-H w_{x x}+u w_{x}\right] d x,
$$

i.e., $w$ should solve the adjoint equation to (33), namely

$$
w_{s}(x, s)=-H w_{x x}(x, s)+u(x, T-s) w_{x}(x, s) .
$$

The time reversal in the inhomogeneous term $u(x, T-s)$ is significant. Combining this with (32) and (34), we conclude that

$$
\frac{\delta G}{\delta u_{0}}(x)=w(x, T)-w_{0}(x)
$$

where $w$ solves (41) with initial condition (35). 
Remark We emphasize that the steps we have just followed for the Benjamin-Ono equation can in principle be carried out for any PDE. These steps are simply:

1. Find the variational equation analogous to (13).

2. Find the appropriate adjoint equation, accounting for time-reversal.

The details of the initial condition of the adjoint problem and the formula for $\frac{\delta G}{\delta u_{0}}$ depend on the particular functional $G$ we choose, but they are usually straightforward to work out. For example, as another variant, we could define

$$
G\left(u_{0}, T\right)=\frac{1}{2} \int_{0}^{2 \pi}[u(x, T / 2)-u(2 \pi-x, T / 2)]^{2} d x
$$

to impose even symmetry at the half-way point. (Recall that if $u_{0}$ is symmetric, then $u(2 \pi-x, T / 2)=u(x,-T / 2)$.) In this case, we find that

$$
\frac{\delta G}{\delta u_{0}}(x)=2 w(x, T / 2), \quad w_{0}(x)=u(x, T / 2)-u(2 \pi-x, T / 2),
$$

or, since $v_{0}$ is assumed symmetric in this formulation, $\frac{\delta G}{\delta u_{0}}(x)=w(x, T / 2)+w(2 \pi-$ $x, T / 2)$. In subsequent work, we will apply the methods of this paper to the vortex sheet with surface tension and to the water wave. Although step 2 usually amounts to a simple integration by parts as was done in (40) above, the adjoint calculation can be quite involved for systems of PDEs with more complicated nonlinearities such as the Birkhoff-Rott integral in the vortex sheet problem (see Ambrose and Wilkening 2009).

\subsection{The Numerical Method}

We minimize $G_{\text {tot }}$ using the BFGS algorithm (Nocedal and Wright 1999), which is a quasi-Newton line search method that builds an approximate Hessian incrementally from the history of gradients it has evaluated. As a black box unconstrained minimization algorithm, it requires only an initial guess and subroutines to compute $G_{\text {tot }}(q)$ and $\nabla_{q} G_{\text {tot }}(q)$, where $q \in \mathbb{R}^{d}$ contains the numerical degrees of freedom used to represent $u_{0}$ and $T$. We use a solution of the linearized problem for the initial guess near a stationary solution or traveling wave, and then use linear extrapolation (or the result of the previous iteration) for the initial guess in subsequent calculations as we vary the bifurcation parameter.

In our implementation, we wrote a C++ wrapper around J. Nocedal's L-BFGS Fortran code released in 1990, but we turn off the limited memory aspect of the code since computing $G$ takes more time than the linear algebra associated with updating the full Hessian matrix. We do find that the algorithm converges quadratically once it gets close to a minimizer. Our code also makes use of the FFTW and LAPACK libraries, but was otherwise written from scratch.

We represent $u(x, t)$ spectrally as a sum of $M$ (typically 384 or 512) Fourier modes,

$$
u(x, t)=\sum_{k=-M / 2+1}^{M / 2} c_{k}(t) e^{i k x}, \quad c_{k} \in \mathbb{C} .
$$


Since $u$ is real, we use the r2c version of the FFT algorithm, which only accesses the coefficients $c_{k}$ with $k \geq 0$, assuming $c_{-k}=\bar{c}_{k}$. We also zero out the Nyquist frequency $c_{M / 2}$ so that the total number of (real) degrees of freedom representing $u$ at time $t$ is $M-1$. We use $d=M / 2$ degrees of freedom to represent $u_{0}$ and $T$, namely

$$
q=\left(a_{0}, T, a_{1}, b_{1}, \ldots, a_{M / 4-1}, b_{M / 4-1}\right) \in \mathbb{R}^{d} \quad\left(c_{k}=a_{k}+i b_{k}, t=0\right) .
$$

The remaining Fourier modes in $u_{0}$ are taken to be zero. The reason for using fewer Fourier modes in the initial condition is that in order to avoid aliasing errors, we want the upper half of the spectrum to remain close to zero throughout the calculation; therefore, we do not wish to give BFGS the opportunity to modify these coefficients. We increase $M$ and repeat the calculation any time one of the high frequency $(k \geq M / 4)$ Fourier modes of the optimal solution exceeds $10^{-13}$ in magnitude at any timestep.

To compute $G(q)$, we write the Benjamin-Ono equation in the form

$$
u_{t}=f(u)+g(u), \quad g(u)=H u_{x x}, f(u)=-\left(\frac{1}{2} u^{2}\right)_{x},
$$

where $\frac{1}{2} u^{2}$ is evaluated on the grid $\left\{x_{j}=2 \pi j / M: 0 \leq j \leq M-1\right\}$ in physical space while $H$ and $\partial_{x}$ are evaluated in Fourier space. The trapezoidal rule in physical space is used to evaluate the integral (30) defining $G$. To evolve the solution, we use the stiffly stable, additive (i.e., implicit-explicit) Runge-Kutta method of Kennedy and Carpenter (Kennedy and Carpenter 2003; Wilkening 2007) known as ARK4(3)6L[2]SA with a fixed timestep $h=T / N$, where $N$ is chosen to be large enough that further refinement does not improve the solution. Briefly, the idea of an ARK method is to treat $f$ explicitly (as it is nonlinear) while treating $g$ implicitly (as it is the source of stiffness):

$$
\begin{array}{lll}
k_{i}=f\left(t_{n}+c_{i} h, u_{n}+h \sum_{j} a_{i j} k_{j}+h \sum_{j} \hat{a}_{i j} \ell_{j}\right), & \\
\ell_{i}=g\left(t_{n}+\hat{c}_{i} h, u_{n}+h \sum_{j} a_{i j} k_{j}+h \sum_{j} \hat{a}_{i j} \ell_{j}\right), & \frac{c \mid A}{b^{T}} & \frac{\hat{c} \mid \hat{A}}{\mid \hat{b}^{T}} \\
u_{n+1}=u_{n}+h \sum_{j} b_{j} k_{j}+h \sum_{j} \hat{b}_{j} \ell_{j} . & \text { for } f
\end{array}
$$

The Butcher array for $f$ satisfies $a_{i j}=0$ if $i \leq j$ and for $g$ satisfies $\hat{a}_{i j}=0$ if $i<j$, which allows the stage derivatives to be solved for in order: $\ell_{1}, k_{1}, \ell_{2}, k_{2}, \ldots, \ell_{6}, k_{6}$, where our scheme has 6 stages. See Kennedy and Carpenter (2003) for the scheme coefficients and Wilkening (2007) for details on solving the implicit equations in the similar cases of Burgers' equation and the $\mathrm{KdV}$ equation.

Once $u(x, T)$ is known, we use the same scheme to solve the adjoint equation

$$
w_{s}=f(s, w)+g(w), \quad g(w)=-H w_{x x}, f(s, w)(x)=u(x, T-s) w_{x}(x) .
$$


The main difficulty is that the intermediate stages of the ARK method require the value of $u$ at intermediate times (between timesteps). For this, we use cubic Hermite interpolation, matching $u$ and $u_{t}$ at the timesteps straddling the required intermediate time:

$$
\begin{aligned}
u\left(\cdot, t_{n}+\theta h\right)= & (1-\theta) u_{n}+\theta u_{n+1}-\theta(1-\theta)\left[(1-2 \theta)\left(u_{n+1}-u_{n}\right)\right. \\
& \left.-(1-\theta) h \partial_{t} u_{n}+\theta h \partial_{t} u_{n+1}\right]
\end{aligned}
$$

where $0<\theta<1$. This yields fourth-order accurate values of $u$ in the right-hand side of (49), which is sufficient to achieve a fourth-order accurate global solution $w$. We include the option in our code to store $u$ only at certain milemarker times, and then regenerate the data at all timesteps between milemarkers as soon as the $w$ equation enters that region; this dramatically reduces the memory requirements of the code at the expense of having to compute $u$ twice.

Once $u(x, T)$ and $w(x, T)$ are known with the period and initial conditions specified in $q \in \mathbb{R}^{d}$, we compute $G(q)$ using the trapezoidal rule in physical space to evaluate the integral in (30), and we compute $\frac{\partial G}{\partial q_{j}}$ by taking the FFT of $\frac{\delta G}{\delta u_{0}}$ and scaling each component appropriately:

$$
\begin{aligned}
\frac{\partial G}{\partial q_{0}} & =\int_{0}^{2 \pi} \frac{\delta G}{\delta u_{0}}(x) 1 d x=2 \pi\left(\frac{\delta G}{\delta u_{0}}\right)_{0}^{\wedge} \\
\frac{\partial G}{\partial q_{1}} & =\frac{\partial G}{\partial T}=\int_{0}^{2 \pi}\left[u(x, T)-u_{0}(x)\right] u_{t}(x, T) d x, \quad \longleftarrow \quad\left(\begin{array}{c}
\text { use trap. rule } \\
\text { in physical space }
\end{array}\right) \\
\frac{\partial G}{\partial q_{2 k}} & =\frac{\partial G}{\partial a_{k}}=\int_{0}^{2 \pi} \frac{\delta G}{\delta u_{0}}(x)\left(e^{i k x}+e^{-i k x}\right) d x=4 \pi \operatorname{Re}\left\{\left(\frac{\delta G}{\delta u_{0}}\right)_{k}^{\wedge}\right\} \quad(k \geq 1), \\
\frac{\partial G}{\partial q_{2 k+1}} & =\frac{\partial G}{\partial b_{k}}=\int_{0}^{2 \pi} \frac{\delta G}{\delta u_{0}}(x)\left(i e^{i k x}-i e^{-i k x}\right) d x=4 \pi \operatorname{Im}\left\{\left(\frac{\delta G}{\delta u_{0}}\right)_{k}^{\wedge}\right\} \quad(k \geq 1) .
\end{aligned}
$$

We remark that these formulas for the derivatives of the numerical version of $G$ essentially assume that we have solved the PDE exactly (so that the calculus of variations applies to our numerical solutions). This is reasonable in our case as we are using spectrally accurate schemes, but would cause difficulties if the numerical solution were only first or second order accurate in space or time.

\subsection{Choice of Penalty Function $\varphi$}

We still need to define the penalty function $\varphi\left(u_{0}, T\right)$ in (29) and show how to compute its gradient with respect to $q$. The purpose of $\varphi$ is to pin down the mean and the phase shifts in space and time as well as to specify the bifurcation parameter. We have explored several successful variants which became more specialized as our understanding of the problem increased. As some of these variants may prove useful in other problems, we describe them here.

Initially, we did not include a penalty function in $G_{\text {tot }}$, but without it the BFGS algorithm invariably converges to a constant solution. Next, we constrained $q_{2}$, the 
real part of the first Fourier mode $\hat{u}_{1}(t)=a_{1}(t)+i b_{1}(t)$ at $t=0$, to have a given value $\rho$. We reasoned that as long as $\rho$ is not too large, the BFGS algorithm can vary $q_{3}=b_{1}(0)$ to find a periodic solution, so all we are doing is pinning down a phase. This was done by defining

$$
\begin{aligned}
& \varphi\left(u_{0}, T\right)=\frac{1}{2}\left(\left[a_{0}(0)-\alpha_{0}\right]^{2}+\left[a_{1}(0)-\rho\right]^{2}\right) \text { or } \\
& \varphi(q)=\frac{1}{2}\left(\left[q_{0}-\alpha_{0}\right]^{2}+\left[q_{2}-\rho\right]^{2}\right),
\end{aligned}
$$

which works well to rule out the constant solutions but generally leads to traveling waves. By studying these traveling waves, we determined the formulas of Sect. 2 and also observed that for some choices of $\rho$ and starting guess $q^{(0)}$, the wave becomes "wobbly," indicating that a nontrivial solution might be nearby.

To rule out traveling waves, we chose a parameter $\eta \in[-1,1]$ and defined

$$
\begin{aligned}
\varphi\left(u_{0}, T\right)= & \frac{1}{2}\left(\left[a_{0}(0)-\alpha_{0}\right]^{2}+\left[a_{1}(0)-\rho\right]^{2}+\left[a_{1}(T / 2)-\eta a_{1}(0)\right]^{2}\right. \\
& \left.+\left[b_{1}(T / 2)-\eta b_{1}(0)\right]^{2}\right) .
\end{aligned}
$$

Our idea here was that a (one-hump) traveling wave would have $\eta= \pm 1$, depending on how many times it passed through the domain in time $T$; hence, intermediate values of $\eta$ would have to correspond to nontrivial solutions. To compute the gradient of $\varphi$ when it involves Fourier modes at later times, we simply solve another adjoint problem. Specifically, if $\varphi$ involves one of

$$
\begin{aligned}
& a_{k}(T / 2)=\frac{1}{2 \pi} \int_{0}^{2 \pi} u(x, T / 2) \cos (k x) d x, \\
& b_{k}(T / 2)=\frac{1}{2 \pi} \int_{0}^{2 \pi} u(x, T / 2)[-\sin (k x)] d x,
\end{aligned}
$$

we will need to compute $\frac{\delta}{\delta u_{0}} a_{k}(T / 2)$ or $\frac{\delta}{\delta u_{0}} b_{k}(T / 2)$, which can be done by setting

$$
w_{0}(x)=\frac{1}{2 \pi} \cos (k x), \quad \text { or } \quad w_{0}(x)=-\frac{1}{2 \pi} \sin (k x)
$$

and solving (41) from $s=0$ to $s=T / 2$; the result $w(x, T / 2)$ is the desired variational derivative. These may then be used to compute $\frac{\partial}{\partial q_{j}} a_{k}(T / 2)$ or $\frac{\partial}{\partial q_{j}} b_{k}(T / 2)$ as was done for $G$ in (50), at which point it is a simple matter to obtain $\frac{\partial \varphi}{\partial q_{j}}$.

This procedure proved very effective in obtaining nontrivial time periodic solutions. The BFGS algorithm is able to minimize $G_{\text {tot }}$ down to $10^{-26}$, at which point roundoff error prevents further reduction. With random initial data $q^{(0)}$ (which we tried before we had solved the eigenvalue problem for the linearization), the algorithm explores quite a wide region of the parameter space, with all components of $q$ (including $T$ ) changing substantially_we do not seem to get stuck in nonzero local 
minima of $G_{\text {tot }}$. Once we do find a nontrivial solution, varying $\eta$ leads to other nearby periodic solutions.

Studying this family of solutions, we finally realized that we were dealing with a four parameter family of nontrivial solutions with the mean, two phases and a bifurcation parameter describing them. The main drawback of using $\eta$ as the bifurcation parameter is that the spatial and temporal phases are not specified independently, but instead depend on $\eta$ in a complicated way. A more natural choice is to define

$$
\varphi\left(u_{0}, T\right)=\frac{1}{2}\left(\left[a_{0}(0)-\alpha_{0}\right]^{2}+\left[a_{k}(0)-\rho\right]^{2}+\left[b_{k}(0)\right]^{2}+\left[\partial_{t} a_{k}(0)\right]^{2}\right),
$$

i.e., we use $\varphi$ to impose the mean $\alpha_{0}$, the bifurcation parameter $\rho$, the spatial phase $b_{k}(0)=0$, and the temporal phase $\partial_{t} a_{k}(0)=0$. Given any solution, we can always translate space and time to achieve the latter two conditions-we have not made any symmetry assumptions here. The index $k$ we use depends on the number of humps $N$ and bifurcation index $n$ of the linearized solution; the only requirement is that $\hat{z}_{N, n, k}$ in (24) be nonzero. One readily checks that

$$
\begin{aligned}
\partial_{t} a_{k}(0) & =\frac{1}{2 \pi} \int_{0}^{2 \pi} u_{t}(x, 0) \cos k x d x \\
& =\frac{1}{2 \pi} \int_{0}^{2 \pi}\left[-k^{2} u_{0}+(k / 2) u_{0}^{2}\right](-\sin k x) d x,
\end{aligned}
$$

from which we obtain $\frac{\delta}{\delta u_{0}}\left[\partial_{t} a_{k}(0)\right](x)=\frac{1}{2 \pi}\left(k^{2}-k u_{0}(x)\right) \sin k x$. Although (51) does not rule out traveling waves, we have no difficulty bifurcating from traveling waves to nontrivial solutions by choosing a starting guess that includes first order corrections from the linear theory of Sect. 3 .

\section{Non-Trivial Time-Periodic Solutions}

We now use the methods described above to study the global behavior of nontrivial time-periodic solutions far beyond the realm of validity of the linearization about stationary and traveling waves. We find that these nontrivial solutions act as rungs in a ladder, connecting stationary and traveling solutions with different speeds and wavelengths by creating or annihilating oscillatory humps that grow or shrink in amplitude until they become part of the stationary or traveling wave on the other side of the rung. The dynamics of these non-trivial solutions are often very interesting, sometimes resembling a low amplitude traveling wave superimposed on a lower frequency carrier signal, and other times behaving like two bouncing solitons that repel each other to avoid coalescing. In this section, we present a detailed numerical study of the path of nontrivial solutions connecting the one-hump stationary solution to the two-hump traveling wave. In Sect. 6, we derive exact formulas for the solutions on this path. In a follow-up paper (Ambrose and Wilkening 2009), we classify all bifurcations from traveling waves, study the paths of nontrivial solutions connecting several of them, and propose a conjecture explaining how they all fit together. 


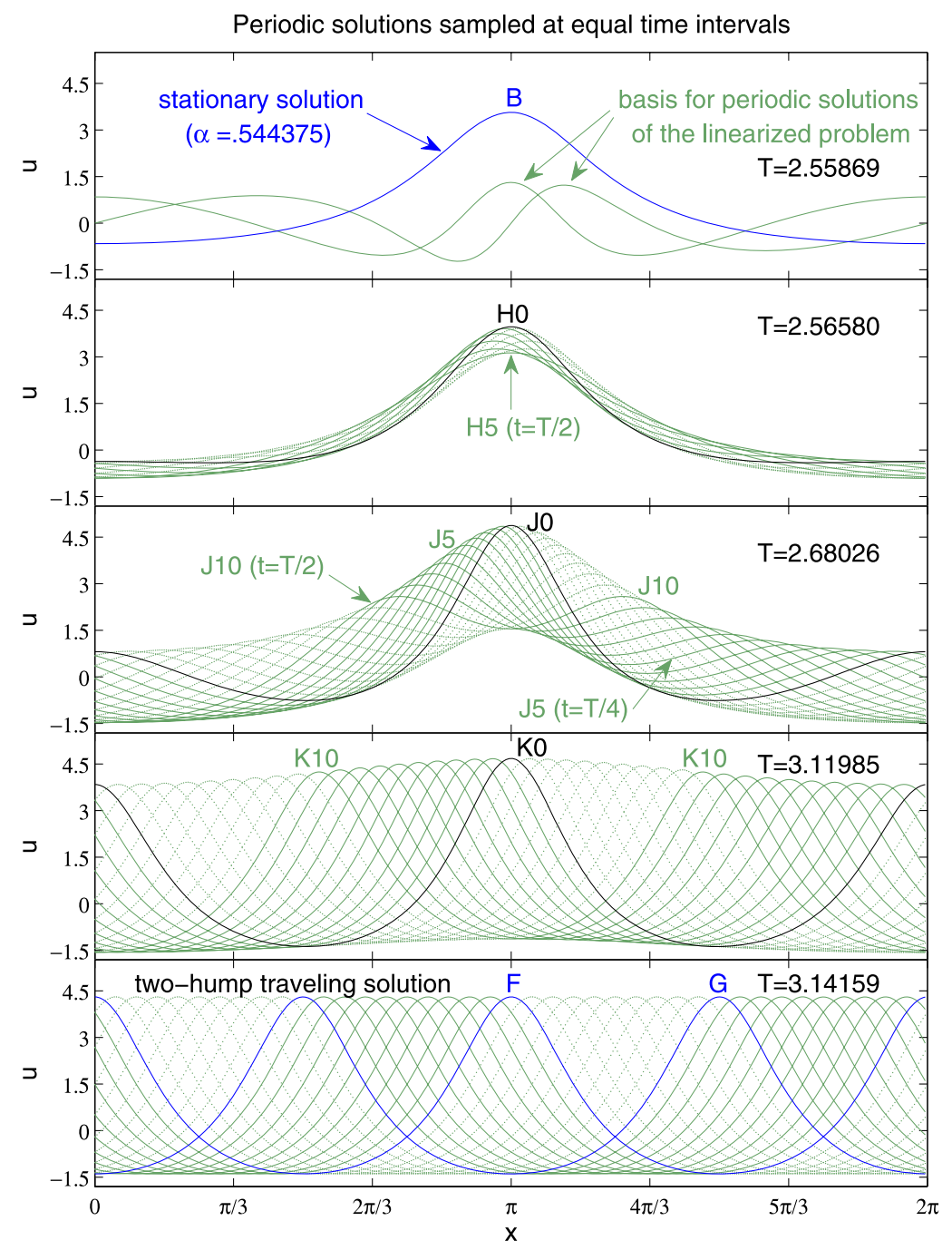

Fig. 3 Progression from a one-hump stationary solution (top) to a two-hump traveling wave (bottom, moving left) by varying the real part of the first Fourier mode at $t=0$ while holding the mean $\alpha_{0}$ constant and choosing the spatial and temporal phases such that $\varphi$ in (51) is zero. The labels B, F, G, H0, J0, etc. correspond to Figs. 4-8

Consider the periodic solutions obtained by bifurcation from the one-hump stationary solution at the lowest frequency, $\omega_{1,1}$. We arbitrarily set the mean $\alpha_{0}=$ 0.544375 for these simulations (see Fig. 1 above), but as shown in Sect. 6, any choice of $\alpha_{0}<1$ would lead to similar results. In the top pane of Fig. 3, we show the onehump stationary solution $u_{1, \beta}(x)$ with $\beta=-\sqrt{\left(1-\alpha_{0}\right) /\left(3-\alpha_{0}\right)}$ together with the (initial conditions of the) two periodic solutions

$$
v^{(0)}(x, t)=\operatorname{Re}\left\{z_{1,1}(x) e^{i \omega_{1} t}\right\}, \quad v^{(1)}(x, t)=\operatorname{Im}\left\{z_{1,1}(x) e^{i \omega_{1} t}\right\}
$$




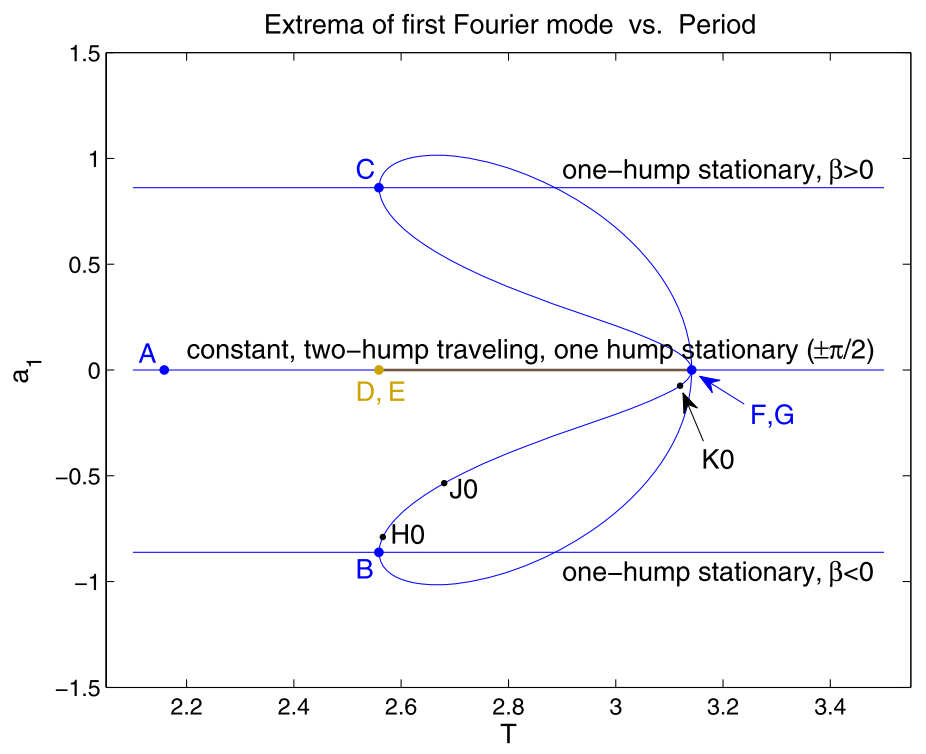

Fig. 4 Bifurcation from one-hump stationary solutions (B and C) to nontrivial time-periodic solutions that reconnect with two-hump traveling waves at $\mathrm{F}$ and $\mathrm{G}$

of the linearized equation (13) corresponding to the first eigenvalue $\omega_{1,1}=3-\alpha_{0}$ of $B A=-i \partial_{x}\left(H \partial_{x}-u\right)$. The natural period of these solutions is $T=2 \pi / \omega_{1,1}=$ 2.55869. Note how the nonlinearity of Benjamin-Ono distorts these two-hump perturbations as they travel (to the left) on top of the one-hump stationary "carrier" solution. Also note that $v^{(0)}$ and $v^{(1)}$ are actually the same solution with a $T / 4$ phase lag in time:

$$
v^{(0)}(x, T / 4)=-v^{(1)}(x, 0) \quad \text { while } \quad v^{(1)}(x, T / 4)=v^{(0)}(x, 0) .
$$

We choose the real part of the first Fourier mode as the bifurcation parameter $\rho$ so that $k=1$ in the definition (51) of $\varphi$. As we vary $\rho=a_{1}(0)$ from $-2 \sqrt{\left(1-\alpha_{0}\right) /\left(3-\alpha_{0}\right)}$ to 0 , we traverse the trajectory from $\mathrm{B}$ to $\mathrm{F}$ in the bifurcation diagram of Fig. 4. The curves corresponding to the intermediate points $\mathrm{H} 0$, J0, and $\mathrm{K} 0$ along this path are shown in black in panels $2-4$ of Fig. 3. Along this path, we see that a second hump forms at $x=0$ while the center hump sharpens to accommodate the shorter wavelength of the two-hump traveling wave. If we instead increase $|\rho|$ near point B in the diagram, we obtain the lower path connecting B to G. Along this path, the center hump decreases in magnitude (curve H5), forms a dimple in the middle (curve J10), splits into two humps (curve K10), and again turns into a two-hump traveling wave (curve $\mathrm{G}$ ). These curves are related to those on the path from B to $\mathrm{F}$ by a $T / 2$ phase shift in time. If we change the sign of $\beta$ (i.e., shift the phase by $\pi$ ) in the stationary solution and call the resulting curve $\mathrm{C}$, the bifurcation diagram is reflected about the $T$ axis. The path from $\mathrm{B}$ (or $\mathrm{C}$ ) to $\mathrm{F}$ is easier to compute due to the turning point in $|\rho|$ on the path from $\mathrm{B}$ (or $\mathrm{C}$ ) to $\mathrm{G}$. 
Fig. 5 The trajectories of the first Fourier mode in the complex plane are exactly circular. The markers on the left lobe correspond to the solutions shown in Fig. 3

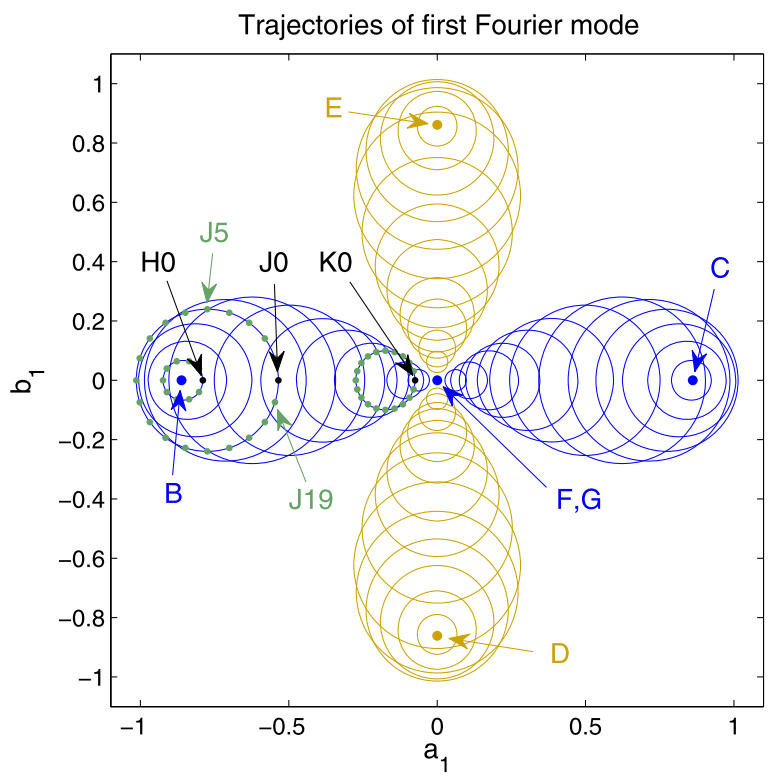

By the time we reach $K 0$ on the path from B to $\mathrm{F}$, we can view our solution as a two-hump traveling wave with a small one-hump stationary perturbation corresponding to the first eigenvalue $\omega_{2,1}=1$ in the linearization about the two-hump traveling wave. A full analysis of the linearization about traveling waves is given in the followup paper (Ambrose and Wilkening 2009), but the idea is that if $u(x)$ is a stationary solution and $U(x, t)=u(x-c t)+c$ is a traveling wave, then the solutions $v$ and $V$ of the linearizations about $u$ and $U$ satisfy $V(x, t)=v(x-c t, t)$. Now, the linearized solutions $\operatorname{Re}\left\{z_{2,1}(x) e^{i \omega_{2,1} t}\right\}$ and $\operatorname{Im}\left\{z_{2,1}(x) e^{i \omega_{2,1} t}\right\}$ about the two-hump stationary solution have the property that $z_{2,1}(x-\pi)=-z_{2,1}(x)$; hence, when they are used as perturbations on a two-hump traveling wave, they need to progress through an extra half-cycle in time to make up for the sign change. As a result, $\omega_{2,1} T$ must belong to $\pi+2 \pi \mathbb{Z}$ (rather than $2 \pi \mathbb{Z}$ itself) for the linearized solution to be periodic. It turns out that as we traverse the path from B to $\mathrm{F}$, the period of the solution increases from $T=2 \pi / \omega_{1,1}$ up to $T=\pi / \omega_{2,1}=\pi$ (rather than e.g. $3 \pi$ or $5 \pi$ ). Note that as $\omega_{2,1}=1$ is independent of $\alpha_{0}$, the path connecting the one hump stationary solution to the two-hump traveling wave always terminates at $T=\pi$, regardless of the mean.

In Fig. 5, we plot the trajectories of the first Fourier mode $c_{1}(t)=a_{1}(t)+i b_{1}(t)$ in the complex plane for various choices of the bifurcation parameter $\rho=a_{1}(0)$. We were surprised to find that these trajectories are exactly circular; this will be discussed further below. The markers on the left (west) lobe of circles correspond to solutions plotted in Fig. 3; for example, J19 corresponds to $u\left(x, \frac{19}{20} T\right)$, which is the dotted curve immediately to the right of the initial condition $\mathrm{J} 0$ in the center pane of Fig. 3. For visibility, we only plotted 10 timeslices in the evolution of $\mathrm{H} 0$.

The four parameter family of nontrivial solutions can be seen in Fig. 5. A given solution is represented by one of the circular trajectories. The two main parameters describing this family are the mean $\alpha_{0}$ and the distance from the nearest point on 
Fig. 6 Bifurcation from the constant solution to a two-hump traveling wave and the path of nontrivial solutions connecting these to various one-hump stationary solutions

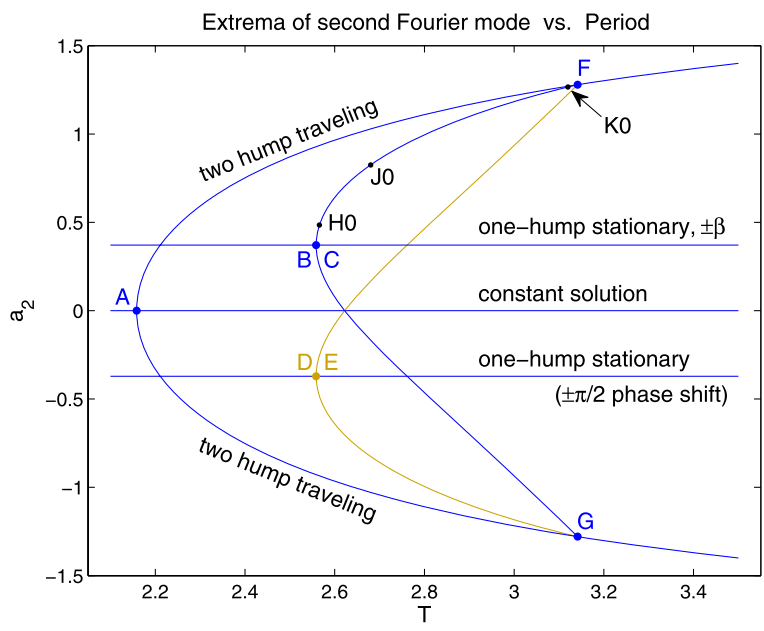

the circle to the origin. A spatial phase shift of the initial condition by $\theta$ (with the sign convention of (22)) amounts to a clockwise rotation of the circle about the origin by $\theta$ (or $k \theta$ for the $k$ th Fourier mode). The north, east, and south lobes of circles represent spatial phase shifts of the west lobe of solutions by $\theta=\pi / 2, \pi$ and $-\pi / 2$, respectively, but any other phase shift $\theta \in \mathbb{R}$ is also allowed. Finally, a temporal phase shift amounts to choosing which point on the circle we assign to $t=0$. Requiring that the initial condition have even symmetry yields either the west or east lobe of solutions with $t=0$ occurring along the real axis.

We can also use other Fourier modes for the bifurcation parameter. This is especially important to track higher order bifurcations from multi-hump traveling waves - in these cases, the first several Fourier modes remain zero for all solutions in the family at all times $t$. But even for the simplest path connecting one-hump stationary solutions to two-hump traveling waves, it is useful to study other bifurcation diagrams representing this same family of solutions. In Fig. 6, we show the result when the second Fourier mode is used instead of the first. By setting $\rho=a_{2}(0)$, we can now also see the bifurcation (labeled A) from the constant solution $u \equiv \alpha_{0}$ to the two-hump traveling waves; moreover, the points $F$ and $G$ that fell on top of each other in Fig. 4 become distinct. The outer curve connecting $F$ to $G$ via A represents the set of two-hump traveling waves moving left with mean $\alpha_{0}$. This curve was plotted parametrically, setting $a_{2}= \pm 2 N \sqrt{(1-\alpha) /(3-\alpha)}$ and $T=2 \pi /\left[N\left(N \alpha-\alpha_{0}\right)\right]$ with $N=2$ and $\alpha$ ranging over all values such that $\alpha \leq 1$ and $T \leq 3.5$.

It is interesting to note that the bifurcation at $F$ (and at $G$ ) from the two-hump traveling wave does not look like a pitchfork. Instead, the bifurcation curve enters at an oblique angle from one side only. This is because the second Fourier mode of the linearized solution $v^{(0)}(x, t)=\operatorname{Re}\left\{z_{2,1}(x+t) e^{i t}\right\}$ is zero (cf. (24) above), so the first order effect on the bifurcation parameter $\rho=a_{2}(0)$ is zero as we move away from the two-hump traveling wave in the direction of $v^{(0)}$. The derivative of $T$ in this direction is also zero, so a heuristic argument based on the Liapunov-Schmidt reduction (Golubitsky and Schaeffer 1985; Kielhöfer 2004) leads to an equation $g(\rho, T)=0$ for the bifurcation curve, where $\frac{\partial g}{\partial T}=0$ and $\frac{\partial g}{\partial \rho}=0$ at the bifurcation. By contrast, the first 
Fig. 7 The trajectories of the second Fourier mode in the complex plane are epitrochoids; see (54). The markers correspond to the solutions plotted in Fig. 3

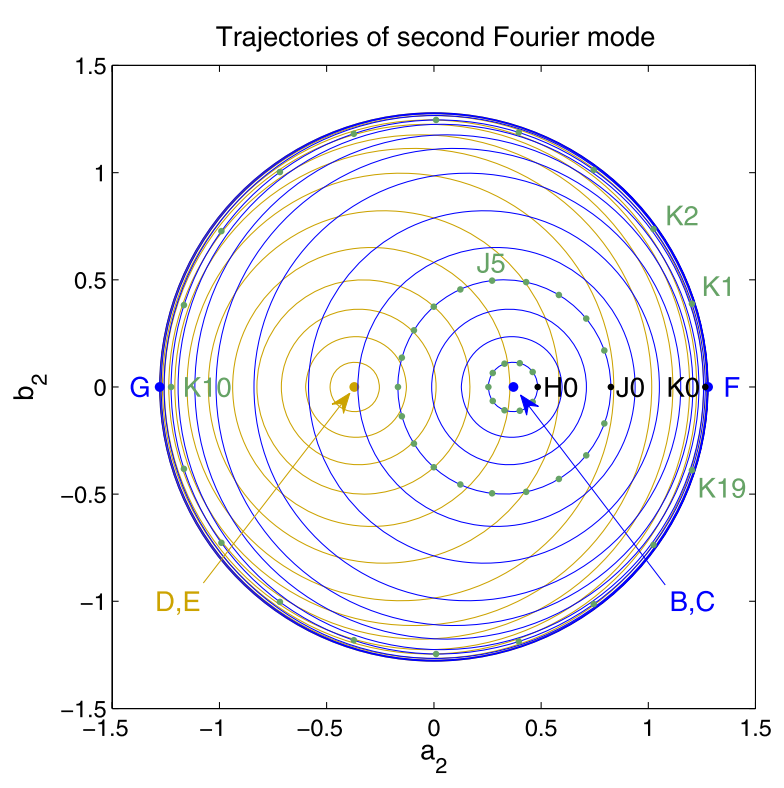

Fourier coefficient of $v^{(0)}(\cdot, 0)$ is nonzero and we do obtain a pitchfork bifurcation at F (and G) when we plot $a_{1}(0)$ vs. $T$, as was seen in Fig. 4.

It turns out that the path of $a_{2}(0)$ vs. $T$ from $\mathrm{F}$ to $\mathrm{B}$ is identical to the one from $\mathrm{F}$ to $\mathrm{C}$; which one-hump stationary solution we end up with depends on whether we perturb the traveling wave in the direction of $+v^{(0)}$ or $-v^{(0)}$. However, there is another direction we can move while keeping $G_{\text {tot }}$ zero (with $k=2$ in (51)), namely $v^{(1)}(x, t)=\operatorname{Im}\left\{z_{2,1}(x+t) e^{i t}\right\}$. This direction breaks the even symmetry of the initial condition, but the even Fourier modes still satisfy $b_{k}(0)=0$ and $\partial_{t} a_{k}(0)=0$; hence, the penalty function $\varphi$ does not exclude this direction when $k=2$ in (51). Depending on whether we perturb in the $+v^{(1)}$ or $-v^{(1)}$ direction, we end up at either the onehump stationary solution $\mathrm{E}$, with maximum at $x=3 \pi / 2$, or $\mathrm{D}$, with maximum at $x=\pi / 2$. This shows that our choice of penalty function $\varphi$ in (51) does not rule out nontrivial solutions with asymmetric initial conditions: the solutions on the path from (F or $\mathrm{G}$ ) to (D or $\mathrm{E}$ ) are all asymmetric at $t=0$; however, these solutions are related to the ones on the path from ( $\mathrm{F}$ or $\mathrm{G})$ to $(\mathrm{B}$ or $\mathrm{C}$ ) by a phase shift in space. We have not found any periodic solutions that cannot be made symmetric at $t=0$ by such a phase shift.

In Fig. 7, we show the trajectories of the second Fourier mode in the complex plane. The markers labeled H0, J0, etc. again correspond to the solutions plotted in Fig. 3. Unlike the first Fourier mode, these trajectories are not exactly circular-but by curve fitting we determined they are epitrochoids, (resembling Ptolemy's model of planetary motion or "spirograph" trajectories), of the form

$$
c_{2}(t)=c_{20}+c_{2,-1} e^{i \omega t}+c_{2,-2} e^{i 2 \omega t} \quad(\omega=2 \pi / T),
$$

where the coefficients $c_{2 j}$ (and $\omega$ ) depend on the bifurcation parameter $\rho$. More generally, by curve fitting our numerical solutions, we have discovered a rather amazing 
property of solutions on this path: the $k$ th Fourier mode is found to be of the form

$$
c_{k}(t)=\sum_{j=-k}^{0} c_{k j} e^{-i j \omega t} \quad(k \geq 0, \omega=2 \pi / T),
$$

where $c_{k j} \in \mathbb{R}$ and $c_{-k}(t)=\overline{c_{k}(t)}$. The general form of solutions on other paths connecting higher order bifurcations is similar, and is described in the follow-up paper (Ambrose and Wilkening 2009). The four parameter family of nontrivial solutions is also nicely represented in this figure, where the parameters are the mean, the furthest point on the epitrochoid, a global rotation about the origin, and the choice of which point on the epitrochoid corresponds to $t=0$. Note that a spatial phase shift of the initial condition by $\theta$ leads to a rotation of a trajectory in this figure clockwise by $2 \theta$, so the north and south lobes of circles in Fig. 5 collapse onto the west family of epitrochoids (around D and E) in Fig. 7 while the west and east lobes of Fig. 5 collapse onto the east family here.

\section{Exact Solutions}

The discovery that the Fourier modes execute Ptolemaic orbits of the form (55) led us to expect that it might be possible to write down the solution in closed form. In this section, we show how to do this for the path of nontrivial solutions connecting the one-hump stationary solution to the two-hump traveling wave. We have now learned of several other methods for finding exact solutions of Benjamin-Ono, notably the bilinear formalism used by Satsuma and Ishimori (1979) and Matsuno (2004) to construct multiperiodic solutions; the reduction by Case (1979) of the ODE (9) to a system shown by Moser to be completely integrable; and the approach of Dobrokhotov and Krichever (1991) using the theory of finite zone integration to construct multiphase solutions. Our approach highlights a feature of these solutions that has not been discussed previously, namely that the Fourier modes of these solutions turn out to be power sums of particle trajectories $\beta_{l}(t)$ in the unit disk $\Delta \subset \mathbb{C}$ whose elementary symmetric functions execute simple circular orbits in the complex plane.

We start with the observation that the meromorphic solutions

$$
u(x, t)=\alpha_{0}+\sum_{l=1}^{N} u_{\beta_{l}(t)}(x), \quad \beta_{l}(t) \in \Delta \text { satisfying (11), }
$$

have the property that the first $N+1$ Fourier modes $c_{k}(t)$ of $u(x, t)$ are closely related to the trajectories of the $\beta_{l}$. Specifically, $\alpha_{0}=c_{0}$ is needed to write down the ODE (11), and we have

$$
\begin{array}{cc}
\beta_{1}(t)+\cdots+\beta_{N}(t)=s_{1}(t), & 2 s_{1}(t)=c_{1}(t), \\
\beta_{1}^{2}(t)+\cdots+\beta_{N}^{2}(t)=s_{2}(t), & 2 s_{2}(t)=c_{2}(t), \\
\cdots & \\
\beta_{1}^{N}(t)+\cdots+\beta_{N}^{N}(t)=s_{N}(t), & 2 s_{N}(t)=c_{N}(t) .
\end{array}
$$


It is a standard theorem of algebra (van der Waerden 1970) that the elementary symmetric functions

$$
\sigma_{j}=\sum_{l_{1}<\cdots<l_{j}} \beta_{l_{1}} \cdots \beta_{l_{j}} \quad(j=1, \ldots, N)
$$

are polynomials in the power sums, e.g.,

$$
\sigma_{0}=1, \quad \sigma_{1}=s_{1}, \quad \sigma_{2}=\frac{s_{1}^{2}-s_{2}}{2}, \quad \sigma_{3}=\frac{s_{1}^{3}-3 s_{1} s_{2}+2 s_{3}}{6} .
$$

The general recurrence relation is

$$
\sigma_{0}=1, \quad \sigma_{j}=\frac{1}{j} \sum_{l=1}^{j}(-1)^{l-1} \sigma_{j-l} s_{l} \quad(j=1, \ldots, N) .
$$

The $\beta_{l}$ are then the zeros of the polynomial

$$
\prod_{l=1}^{N}\left(z-\beta_{l}(t)\right)=\sum_{j=0}^{N}(-1)^{j} \sigma_{j}(t) z^{N-j}
$$

We can test whether a given numerical solution $u(x, t)$ is an $N$-particle meromorphic solution by computing its first $N+1$ Fourier coefficients $c_{k}(0)=2 s_{k}(0)$, using (60) to obtain the symmetric functions $\sigma_{j}(0)$, solving for the roots $\beta_{l}(0)$ of the polynomial on the right-hand side of (61), and checking that higher power sums do in fact agree with the Fourier coefficients of the solution:

$$
\beta_{1}^{k}(0)+\cdots+\beta_{N}^{k}(0)=\frac{1}{2} c_{k}(0) \quad(k \geq N+1) .
$$

Using this approach, we find (numerically) that the solutions on the path connecting the one-hump stationary solution to the two-hump traveling wave are 2-particle solutions. Moreover, the trajectories of the first two symmetric functions appear to be of the form

$$
\begin{aligned}
& \sigma_{1}=\beta_{1}+\beta_{2}=-A+B e^{i \omega t}, \\
& \sigma_{2}=\beta_{1} \beta_{2}=-C e^{i \omega t},
\end{aligned}
$$

where $A, B, C, \omega$ are positive constants; see Fig. 8. We now prove this rigorously.

Theorem 1 There is a four-parameter family of time-periodic, two-particle solutions of the form

$$
u(x, t)=\alpha_{0}+u_{\beta_{1}(t)}(x)+u_{\beta_{2}(t)}(x),
$$

where $\beta_{1}(t)$ and $\beta_{2}(t)$ are the roots of the equation

$$
z^{2}-\sigma_{1}(t) z+\sigma_{2}(t)=0
$$



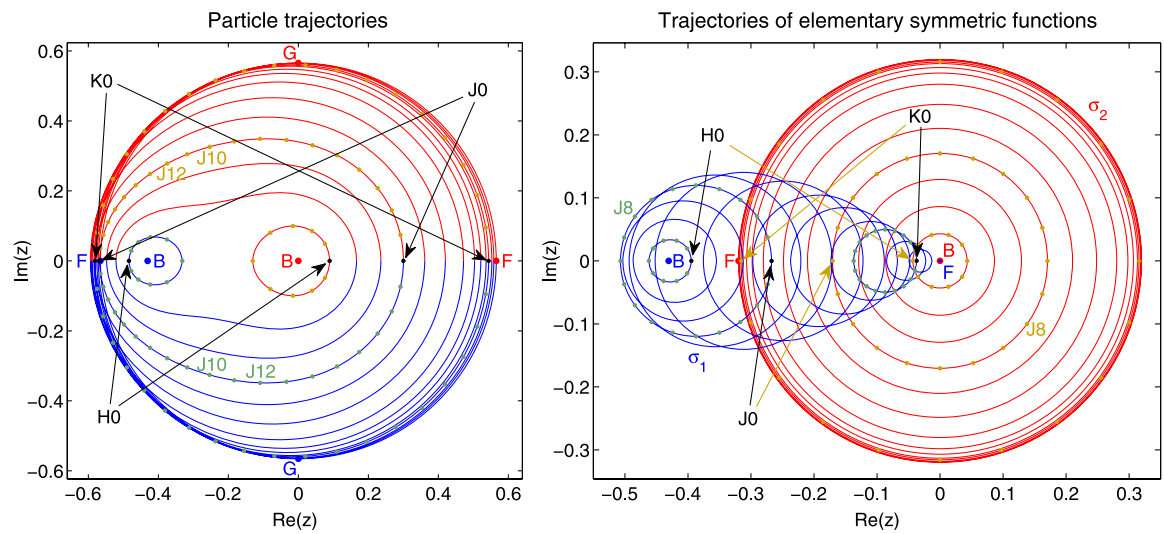

Fig. 8 Left: trajectories of $\beta_{1}, \beta_{2}$ for the solutions in Figs. 3-7. As we vary the bifurcation parameter, the trajectories change from two disjoint, counterclockwise loops to one larger orbit in which $\beta_{1}, \beta_{2}$ exchange positions over the course of one period. Right: the trajectories of $\sigma_{1}, \sigma_{2}$ are exactly circular

and

$$
\begin{aligned}
\sigma_{1}(t) & =\left[-A+B e^{i \omega\left(t-t_{0}\right)}\right] e^{-i \theta}, \quad \sigma_{2}(t)=\left[-C e^{i \omega\left(t-t_{0}\right)}\right] e^{-2 i \theta}, \\
A & =\frac{\left(3-\alpha_{0}\right) \sqrt{\left[\left(3-\alpha_{0}\right)-\left(7-\alpha_{0}\right) C^{2}\right]\left[\left(1-\alpha_{0}\right)-\left(5-\alpha_{0}\right) C^{2}\right]}}{\left(3-\alpha_{0}\right)^{2}-\left(5-\alpha_{0}\right)^{2} C^{2}}, \\
B & =\frac{5-\alpha_{0}}{3-\alpha_{0}} A C, \\
\omega & =\frac{\left(3-\alpha_{0}\right)^{2}-\left(5-\alpha_{0}\right)^{2} C^{2}}{\left(3-\alpha_{0}\right)-\left(5-\alpha_{0}\right) C^{2}} .
\end{aligned}
$$

The four parameters are the mean $\alpha_{0}<1$, two phases $\theta, t_{0} \in \mathbb{R}$, and a real number $C$ ranging from $C=0$ (at the one-hump stationary solution) to $C=\sqrt{\frac{1-\alpha_{0}}{5-\alpha_{0}}}$ (at the two-hump traveling wave).

Proof It suffices to consider the case that $\theta=0$ and $t_{0}=0$ as the general case follows immediately. If we try to substitute $\beta_{1,2}=\frac{\sigma_{1}}{2} \pm \frac{1}{2} \sqrt{\sigma_{1}^{2}-4 \sigma_{2}}$ into the system

$$
\begin{gathered}
\dot{\beta}_{1}=\frac{-2 i \beta_{1}^{2}}{\beta_{1}-\beta_{2}}+\frac{2 i \beta_{1}^{2}}{\beta_{1}-\bar{\beta}_{1}^{-1}}+\frac{2 i \beta_{1}^{2}}{\beta_{1}-\bar{\beta}_{2}^{-1}}+i\left(3-\alpha_{0}\right) \beta_{1}, \\
\dot{\beta}_{2}=\frac{-2 i \beta_{2}^{2}}{\beta_{2}-\beta_{1}}+\frac{2 i \beta_{2}^{2}}{\beta_{2}-\bar{\beta}_{1}^{-1}}+\frac{2 i \beta_{2}^{2}}{\beta_{2}-\bar{\beta}_{2}^{-1}}+i\left(3-\alpha_{0}\right) \beta_{2}
\end{gathered}
$$


and solve for $A, B$, and $\omega$ in terms of $C$ and $\alpha_{0}$, the algebra becomes unmanageable. However, we can rewrite this system in terms of $\sigma_{1}$ and $\sigma_{2}$ to obtain

$$
\begin{aligned}
& \dot{\sigma}_{1}=-2 i\left\{\frac{\beta_{1}\left(\beta_{1} \bar{\beta}_{1}\right)}{1-\beta_{1} \bar{\beta}_{1}}+\frac{\beta_{1}\left(\beta_{1} \bar{\beta}_{2}\right)}{1-\beta_{1} \bar{\beta}_{2}}+\frac{\beta_{2}\left(\beta_{2} \bar{\beta}_{1}\right)}{1-\beta_{2} \bar{\beta}_{1}}+\frac{\beta_{2}\left(\beta_{2} \bar{\beta}_{2}\right)}{1-\beta_{2} \bar{\beta}_{2}}\right\}+i\left(1-\alpha_{0}\right) \sigma_{1}, \\
& \dot{\sigma}_{2}=-2 i\left\{\frac{\beta_{1} \bar{\beta}_{1}}{1-\beta_{1} \bar{\beta}_{1}}+\frac{\beta_{1} \bar{\beta}_{2}}{1-\beta_{1} \bar{\beta}_{2}}+\frac{\beta_{2} \bar{\beta}_{1}}{1-\beta_{2} \bar{\beta}_{1}}+\frac{\beta_{2} \bar{\beta}_{2}}{1-\beta_{2} \bar{\beta}_{2}}\right\} \sigma_{2}+2 i\left(2-\alpha_{0}\right) \sigma_{2} .
\end{aligned}
$$

The expressions inside braces remain invariant if we interchange $\beta_{1}$ and $\beta_{2}$; hence, they may be written as rational functions of $\sigma_{1}, \sigma_{2}, \bar{\sigma}_{1}, \bar{\sigma}_{2}$. Explicitly, we have

$$
\begin{aligned}
\dot{\sigma}_{1}= & -2 i \frac{P_{1}}{Q}+i\left(1-\alpha_{0}\right) \sigma_{1}, \quad \dot{\sigma}_{2}=-2 i \frac{P_{2}}{Q} \sigma_{2}+2 i\left(2-\alpha_{0}\right) \sigma_{2}, \\
P_{1}= & \sigma_{1}^{2} \bar{\sigma}_{1}-2 \bar{\sigma}_{1} \sigma_{2}-2 \sigma_{1}^{3} \bar{\sigma}_{2}+6 \sigma_{1}\left|\sigma_{2}\right|^{2}-\sigma_{1} \bar{\sigma}_{1}^{2} \sigma_{2}+2 \sigma_{1}^{2} \bar{\sigma}_{1}\left|\sigma_{2}\right|^{2} \\
& -2 \bar{\sigma}_{1} \sigma_{2}^{2} \bar{\sigma}_{2}-2 \sigma_{1}\left|\sigma_{2}\right|^{4}, \\
P_{2}= & \left|\sigma_{1}\right|^{2}\left(1+3\left|\sigma_{2}\right|^{2}\right)+4\left|\sigma_{2}\right|^{2}\left(1-\left|\sigma_{2}\right|^{2}\right)-2\left(\sigma_{1}^{2} \bar{\sigma}_{2}+\bar{\sigma}_{1}^{2} \sigma_{2}\right), \\
Q= & \left(1-\left|\sigma_{2}\right|^{2}\right)^{2}-\left|\sigma_{1}\right|^{2}\left(1+\left|\sigma_{2}\right|^{2}\right)+\left(\sigma_{1}^{2} \bar{\sigma}_{2}+\bar{\sigma}_{1}^{2} \sigma_{2}\right) .
\end{aligned}
$$

Since $Q$ is a product of non-zero terms of the form $\left(1-\beta_{i} \bar{\beta}_{j}\right)$, it is never zero. If we assume $\sigma_{1}=-A+B e^{i \omega t}, \sigma_{2}=-C e^{i \omega t}$, and $C \neq 0$, we find that (73) holds as long as

$$
\begin{aligned}
{\left[-2 P_{1}+\left(1-\alpha_{0}\right) \sigma_{1} Q\right]+\frac{B}{C}\left[-2 P_{2} \sigma_{2}+\left(4-2 \alpha_{0}\right) \sigma_{2} Q\right] } & =0 \\
-2 P_{2}+\left(4-2 \alpha_{0}-\omega\right) Q & =0 .
\end{aligned}
$$

We eliminated $\omega$ in (74) using $\dot{\sigma}_{1}=i \omega B e^{i \omega t}=-\frac{B}{C} \dot{\sigma}_{2}$. Next, we collect terms containing like powers of $e^{i \omega t}$ and set them each to zero. This yields 7 polynomial equations in the variables $A, B, C, \alpha_{0}$, and $\omega$; however, several of them are redundant due to relationships such as $Q^{(-1)}=Q^{(1)}$ in the decomposition $Q=$ $Q^{(-1)} e^{-i \omega t}+Q^{(0)}+Q^{(1)} e^{i \omega t}$. Equation (74) yields 4 such equations; two of them are satisfied if we choose $B$ as in (69) while the remaining two are satisfied if we also choose $A$ as in (68). With these choices, all three equations associated with (75) are satisfied provided $\omega$ satisfies (70). The special cases $\left\{C=0, A=\sqrt{\frac{1-\alpha_{0}}{3-\alpha_{0}}}, B=0\right\}$ and $\left\{C=\sqrt{\frac{1-\alpha_{0}}{5-\alpha_{0}}}, A=0, B=0\right\}$ are seen to correspond to the one-hump stationary solution and two-hump traveling wave, respectively, as discussed in Sect. 2.

We have verified that the curve connecting $\mathrm{B}$ to $\mathrm{F}$ in the bifurcation diagram of Fig. 4 is recovered if we set $\alpha_{0}=.544375$ and plot $2(-A+B)$ versus $T=2 \pi / \omega$ using the above formulas for $A, B$ and $\omega$ with $C$ ranging from 0 to $\sqrt{\frac{1-\alpha_{0}}{5-\alpha_{0}}}$. 


\section{Conclusion}

We have presented a general method for finding continua of time-periodic solutions for nonlinear systems of partial differential equations. We have used our method to study global paths of nontrivial time-periodic solutions connecting stationary and traveling waves of the Benjamin-Ono equation. In spite of the non-linearity and nonlocality of the Benjamin-Ono equation, these nontrivial solutions can be interpreted as distorted superpositions of the stationary or traveling waves at each end of the path. Our numerical method is accurate enough that we are able to use data fitting techniques to recognize the analytical form of the solutions. In particular, the Fourier coefficients $c_{k}(t)$ of these solutions follow Ptolemaic orbits of the form (55). This led us to reformulate the equations governing meromorphic pole dynamics to reveal an exact formula for the solutions on the four-parameter path connecting the one-hump stationary solution to the two-hump traveling wave.

In the future, we plan to apply this method to more complicated systems arising in fluid dynamics, namely the vortex sheet and water wave problems. This will allow for comparison with prior numerical and analytical results (Hou et al. 1997; Plotnikov and Toland 2001; Iooss et al. 2005). Additionally, as the Benjamin-Ono equation is meant as a model for internal waves in a deep, stratified fluid, it will be of interest to compare time-periodic vortex sheets and water waves with time-periodic solutions of Benjamin-Ono.

Open Access This article is distributed under the terms of the Creative Commons Attribution Noncommercial License which permits any noncommercial use, distribution, and reproduction in any medium, provided the original author(s) and source are credited.

\section{References}

Ambrose, D.M., Wilkening, J.: Global paths of time-periodic solutions of the Benjamin-Ono equation connecting pairs of traveling waves. Commun. Appl. Math. Comput. Sci. 4, 177-215 (2009)

Ambrose, D.M., Wilkening, J.: Computation of symmetric, time-periodic solutions of the vortex sheet with surface tension. Proc. Natl. Acad. Sci. USA (2010, in press)

Arenstorf, R.F.: Periodic solutions of the restricted three body problem representing analytic continuations of Keplerian elliptic motions. Am. J. Math. LXXXV, 27-35 (1963)

Benjamin, T.B.: Internal waves of permanent form in fluids of great depth. J. Fluid Mech. 29(3), 559-592 (1967)

Brezis, H.: Periodic solutions of nonlinear vibrating strings and duality principles. Bull. Am. Math. Soc. (N.S.) 8, 409-426 (1983)

Bristeau, M.O., Glowinski, R., Périaux, J.: Controllability methods for the computation of time-periodic solutions; application to scattering. J. Comput. Phys. 147, 265-292 (1998)

Brown, H.S., Kevrekidis, I.G., Oron, A., Rosenau, P.: Bifurcations and pattern formation in the "regularized" Kuramoto-Sivashinsky equation. Phys. Lett. A 163, 299-308 (1992)

Broyden, C.G.: The convergence of a class of double-rank minimization algorithms, Parts I and II. J. Inst. Math. Appl. 6, 76-90, 222-231 (1970)

Cabral, M., Rosa, R.: Chaos for a damped and forced KdV equation. Physica D 192, 265-278 (2004)

Case, K.M.: The N-soliton solution of the Benjamin-Ono equation. Proc. Natl. Acad. Sci. USA 75(8), 3562-3563 (1978)

Case, K.M.: Meromorphic solutions of the Benjamin-Ono equation. Physica A 96, 173-182 (1979)

Chen, M., Iooss, G.: Standing waves for a two-way model system for water waves. Eur. J. Mech. B/Fluids 24, 113-124 (2005) 
Cooper, G.J., Sayfy, A.: Additive Runge-Kutta methods for stiff ordinary differential equations. Math. Comput. 40(161), 207-218 (1983)

Crannell, A.: The existence of many periodic non-travelling solutions to the Boussinesq equation. J. Differ. Equ. 126, 169-183 (1996)

Davis, R.E., Acrivos, A.: Solitary internal waves in deep fluid. J. Fluid Mech. 29, 593-607 (1967)

Dobrokhotov, S.Yu., Krichever, I.M.: Multi-phase solutions of the Benjamin-Ono equation and their averaging. Math. Notes 49, 583-594 (1991)

Doedel, E.J., Keller, H.B., Kernévez, J.P.: Numerical analysis and control of bifurcation problems: (I) Bifurcation in finite dimensions. Int. J. Bifurc. Chaos 1(3), 493-520 (1991)

Doedel, E.J., Keller, H.B., Kernévez, J.P.: Numerical analysis and control of bifurcation problems: (II) Bifurcation in infinite dimensions. Int. J. Bifurc. Chaos 1(4), 745-772 (1991)

Duistermaat, J.J.: Bifurcations of periodic solutions near equilibrium points of Hamiltonian systems. In: Bifurcation Theory and Applications. Lecture Notes in Mathematics, vol. 1057, pp. 57-105. Springer, Berlin (1984)

Field, R.J., Burger, M.: Oscillations and Traveling Waves in Chemical Systems. Wiley, New York (1985)

Ginibre, J., Velo, G.: Smoothing properties and existence of solutions for the generalized Benjamin-Ono equations. J. Differ. Equ. 93, 150-212 (1991)

Glowinski, R., Rossi, T.: A mixed formulation and exact controllability approach for the computation of the periodic solutions of the scalar wave equation. (I): Controllability problem formulation and related iterative solution. C. R. Acad. Sci. Paris, Ser. I 343, 493-498 (2006)

Golubitsky, M., Schaeffer, D.G.: Singularities and Groups in Bifurcation Theory, vols. I and II. Springer, New York (1985)

Govaerts, W.J.F.: Numerical Methods for Bifurcations of Dynamical Equilibria. SIAM, Philadelphia (2000)

Hairer, E., Norsett, S.P., Wanner, G.: Solving Ordinary Differential Equations I: Nonstiff Problems, 2nd edn. Springer, Berlin (2000)

Hou, T., Lowengrub, J., Shelley, M.: Removing the stiffness from interfacial flows with surface tension. J. Comput. Phys. 114(2), 312-338 (1994)

Hou, T., Lowengrub, J., Shelley, M.: The long-time motion of vortex sheets with surface tension. Phys. Fluids 9(7), 1933-1954 (1997)

Iooss, G., Plotnikov, P.I., Toland, J.F.: Standing waves on an infinitely deep perfect fluid under gravity. Arch. Ration. Mech. Anal. 177, 367-478 (2005)

Kennedy, C.A., Carpenter, M.H.: Additive Runge-Kutta schemes for convection-diffusion-reaction equations. Appl. Numer. Math. 44(1-2), 139-181 (2003)

Kielhöfer, H.: Bifurcation Theory: An Introduction with Applications to PDEs. Springer, New York (2004)

Lust, K., Roose, D.: Newton-Picard methods with subspace iteration for computing periodic solutions of partial differential equations. Z. Angew. Math. Mech. 76(S2), 605-606 (1996)

Matsuno, Y.: New representations of multiperiodic and multisoliton solutions for a class of nonlocal soliton equations. J. Phys. Soc. Jpn. 73(12), 3285-3293 (2004)

Mawhin, J.: Periodic solutions of some semilinear wave equations and systems: A survey. Chaos Solitons Fractals, 1651-1669 (1995)

Nirenberg, L.: Topics in Nonlinear Functional Analysis. American Mathematical Society, Providence (2001)

Nocedal, J., Wright, S.J.: Numerical Optimization. Springer, New York (1999)

Ono, H.: Algebraic solitary waves in stratified fluids. J. Phys. Soc. Jpn. 39, 1082-1091 (1975)

Pao, C.V.: Numerical methods for time-periodic solutions of nonlinear parabolic boundary value problems. SIAM J. Numer. Anal. 647-667 (2001)

Pironeau, O.: Optimal Shape Design for Elliptic Systems. Springer, New York (1984)

Plotnikov, P.I., Toland, J.F.: Nash-Moser theory for standing water waves. Arch. Ration. Mech. Anal. 159, 1-83 (2001)

Rabinowitz, P.H.: Periodic solutions of Hamiltonian systems. Commun. Pure Appl. Math. 31, 157-184 (1978)

Rabinowitz, P.H.: Periodic solutions of Hamiltonian systems: A survey. SIAM J. Math. Anal. 13, 343-352 (1982)

Satsuma, J., Ishimori, Y.: Periodic wave and rational soliton solutions of the Benjamin-Ono equation. J. Phys. Soc. Jpn. 46(2), 681-687 (1979)

Saut, J.C.: Sur quelques generalisations de l'equation de Korteweg-deVries. J. Math. Pures Appl. 58, 21$61(1979)$ 
Smiley, M.W.: Breathers and forced oscillations of nonlinear wave equations on $\mathbf{R}^{3}$. J. Reine Angew. Math. 398, 25-35 (1989)

Smiley, M.W.: Numerical determination of breathers and forced oscillations of nonlinear wave equations. In: Computational Solution of Nonlinear System of Equations. Lectures in Appl. Math., vol. 26, pp. 605-617. American Mathematical Society, Providence (1990)

Stoer, J., Bulirsch, R.: Introduction to Numerical Analysis, 3rd edn. Springer, New York (2002)

Strogatz, S.H.: Nonlinear Dynamics and Chaos. Perseus Books Group (2000)

Tao, T.: Global well-posedness for the Benjamin-Ono equation in $H^{1}(\mathbf{R})$. J. Hyperbolic Differ. Equ. 1, 27-49 (2004)

van der Waerden, B.L.: Algebra, vol. 1. Unger, New York (1970)

Wilkening, J.: An algorithm for computing Jordan chains and inverting analytic matrix functions. Linear Algebra Appl. 427, 6-25 (2007)

Wilkening, J.: Math 228A Lecture Notes: Numerical Solution of Differential Equations. UC Berkeley (2007). Available from author's webpage

Zehnder, E.: Periodic solutions of Hamiltonian equations. In: Dynamics and Processes. Lecture Notes in Mathematics, vol. 1031, pp. 172-213. Springer, Berlin (1983) 